\title{
Application of eddy-viscosity turbulence models to problems in ship hydrodynamics
}

\author{
Momchil Terziev $^{1 *}$, Tahsin Tezdogan ${ }^{1}$, Atilla Incecik ${ }^{2}$ \\ ${ }^{1}$ Department of Naval Architecture, Ocean and Marine Engineering, Henry Dyer Building, \\ University of Strathclyde, 100 Montrose Street, Glasgow G4 OLZ, UK \\ ${ }^{2}$ University of Strathclyde, Faculty of Engineering, Glasgow, UK \\ *Corresponding author: momchil.terziev@strath.ac.uk
}

\begin{abstract}
With the rapid advent of computational methods in all fields of engineering, several areas have emerged as significant sources of ambiguity. Among these is the selection of a turbulence model to close the Reynolds averaged Navier-Stokes equation. In ship hydrodynamics, this has been particularly difficult to resolve due to the complex nature of the problem. Furthermore, there are a wide variety of turbulence models all claiming superiority. Thus, navigating to the correct choice is a subject of experience. The present study aims to alleviate the ambiguity inherent in the field. This is done by performing a series of tests on the turbulence models and comparing the integral outcomes with experimental results. Specifically, shallow water cases are chosen due to the additional layer of complexity associated in the prediction of parameters of interest. The results are analysed via a modified bivariate plot, which reveals a strong candidate for the optimum choice of turbulence modelling. The assessment simultaneously takes into account resistance and sinkage, in addition to consistency across different casestudies. The time per iteration also points towards the same candidate, identified as the standard $k$ - $\omega$ model, as a good choice within the software used to perform the analysis. The results also suggest that pressure resistance and its constituent components are not coupled with the turbulence model. On the other hand, frictional resistance is highly dependent on the closure selected and is identified as the main contributor to deviations with regards to experimental values.
\end{abstract}

\section{Introduction}

Since the beginning of the $19^{\text {th }}$ century, the fields of science and engineering have been underpinned by the concept of determinism. Its principle was first articulated by Pierre-Simon Laplace (van Strien, 2014) to express the idea that every event is causally determined by previous occurrences. Therefore, if we possess sufficient information about these causes, it is possible to predict the state of a system at any point in the future. This idea likely feels natural to engineers in particular.

Mathematically, determinism is merely an expression that a unique solution of the NavierStokes equations exists. In fact, this has been shown to be the case in two dimensions, but for three-dimensional cases it holds for finite times only (Lesieur, 2008). In other words, it is not 
always possible to obtain sufficient knowledge of antecedent events in order to determine the exact state of the system at some future time. In practice, the engineer is rarely, if ever, interested in the level of detail referred to above. Hence, the use of statistical modelling of physical processes is justified as long as an accurate mean, or integral, value can be obtained.

The question then shifts from our ability to capture the physical interaction of every molecule of the fluid, to its overall properties, such as velocity and pressure. In other words, the fluid is treated as a continuum. The difficulty here is associated with the complexity of turbulent phenomena. Although we observe these in our everyday lives, modelling exact statistical averages of fluctuations inherent in turbulence has proven impossible (Durbin and Pettersson Reif, 2011). Thus, every time the numerical solution to a problem involving turbulent properties is invoked, it contains a degree of empiricism. This empiricism is required, due to the averaging process, whether temporally or spatially, applied to the Navier-Stokes equations. Upon performing what is known as Reynolds averaging, additional terms are introduced as a consequence of nonlinearities in the governing equations. Perhaps surprisingly, the degree of empiricism (the number of empirically defined coefficients, or lack thereof) is not always an indication of accuracy.

The addition of new unknowns to the governing equations means that the Reynolds Averaged Navier-Stokes (RANS) equations no longer form a closed set. In simple terms, there are now more unknowns than there are equations, which is why turbulence modelling is also referred to as providing closure. The additional (partial differential) equations introduced define the type of turbulence model. Hence, we will refer to zero, one, or two-equation closures. Now, we can safely shift the topic of investigation to identifying which turbulence model is optimal for the particular case it is applied to. The optimal solution must exhibit several characteristics:

1. The solution must lie within some predefined measure of accuracy.

2. The effort required to obtain the solution must not be excessive.

3. There should be no simpler method to achieve the same, or reasonably similar result.

In most cases, one can gain an idea of the expected level of accuracy depending on the method used. For example, it is well-known that linear, inviscid potential flow theories do not perform well in extreme cases, where nonlinear phenomena dominate. However, the above (or any other) classification of turbulence models, cannot be used to establish such a hierarchy. Indeed, one-equation models can, and do perform better than two equation models in certain cases. This is true because the derivation of most of the currently available models are not rooted in the physics of the problem per se (Argyropoulos and Markatos, 2015). Simply put, some of the empirical constants contained within the turbulence models are determined by fitting data to simple experimental observations. There is no telling what their effect would be if they were applied to a different problem, as is often the case. Moreover, the mathematical formulation of the equations used to provide closure are rarely related to the physics of the problem either (Markatos, 1986).

The present paper is addressed to practitioners as well as researchers of RANS-based ship hydrodynamic analysis, with the goal of reducing some of the ambiguity inherent in the field. We aim to analyse the issue of optimal turbulence model selection as applied to problems pertaining to the realm of ship hydrodynamics. Complex problems in the field are sought to test the turbulence models robustly. To elaborate, it would be counterproductive to select a simple, two dimensional case since the aim is not to improve on the selected models. The 
parameters of interest are ship bare hull resistance, sinkage, and trim, due to the difficulties associated in with their prediction. Furthermore, Larsson et al., (2014) reported a scatted in sinkage and trim results when examining submissions to the Gothenburg numerical ship hydrodynamics workshop, justifying the above selections. Deliberately accentuating the effect each of these has on the remaining parameters as well as their overall importance implies the selection of a shallow water case-study.

In shallow water, the sinkage, trim, and resistance are influenced by the adverse pressure gradient acting longitudinally on the hull, generated as a result of the hull's proximity with the seabed. Additionally, in terms of turbulence, pressure gradients present an extra layer of complexity, superimposed of the already difficult problems of predicting highly three dimensional boundary layers inherent in ship underwater shapes. For this study, the hull form selected is the benchmark KCS container ship, while the analysis is performed using Star$\mathrm{CCM}+$, version 13.01.011.

The remainder of this work will continue with an overview and identification of selected turbulence models. Then, the case-studies and numerical implementation are presented in Section 3 and 4. Results and their analysis follows form these in Section 5, while conclusions and future work recommendations are given in Section 6.

\section{Background}

The present study is motivated partially by the findings of Larsson et al., (2014), who observed a scatter in sinkage and trim predictions, as alluded to previously, and partially by recent work undertaken by the present authors. Sinkage and trim proved more difficult to simulate accurately than resistance in CFD (Terziev et al., 2019). In deep waters, the combined effect of sinkage and trim is known to be small on resistance and in some cases may even be neglected (Ponkratov, 2016). However, their magnitude and influence is accentuated by the proximity of the seabed in shallow water cases. This not only represents a case where the underlying physics are not well understood, but also, an everyday situation all ships must cope with. More importantly, groundings, where the combined effect of sinkage and trim (termed ship squat) play a central role, are consistently in the top five incident categories of EMSA (European Maritime Safety Agency, 2018, 2017, 2016, 2015) since records began in 2011. In fact, the annual figures published by EMSA suggest more than half of all incidents occur in shallow water, where ship squat is always a contributing factor. Thus, the accurate prediction of ship squat is of critical importance.

While ship squat is a phenomenon requiring further research, turbulence modelling is a 'known unknown', which contributes to the present levels of ambiguity inherent in shallow water predictions. Greater levels of attention are typically attributed to the prediction of resistance. However, there is less ambiguity in its predictions. This has led to rule of thumb approaches even in CFD by international bodies, such as the International Towing Tank Conference (ITTC, 2014). On the other hand, sinkage and trim have proved more evasive. The results from the present research will enable generalisations to be carried into deep water cases for enhanced predictions, even when running trim and sinkage are of little importance. The remainder of this section will proceed by examining the turbulence models. 


\subsection{Turbulence}

Attempts to bring turbulence under mathematical scrutiny begin with Boussinesq's concept of eddy-viscosity (Wilcox, 2006), which he developed from ideas expressed by Saint-Venant (Darrigol, 2017). This makes it possible to express the Reynolds stress tensor, which is the additional term introduced in the process of Reynolds averaging, as a function of mean flow properties. Here, Reynolds averaging refers to the process of averaging the decomposed total velocity (mean and fluctuating parts) (Durbin and Pettersson Reif, 2011). The eddy-viscosity is a property of the flow, i.e. a function of space and time, unlike the molecular viscosity, which is an intrinsic physical constant (Bailly and Comte-Bellot, 2004).

The first turbulence model was introduced by Prandtl (1925) using the mixing length concept to compute the eddy-viscosity. This model did not contain any Partial Differential Equations (PDEs), and is therefore known as zero-equation or algebraic. In practice, an n-equation model refers to the number of additional PDEs introduced to close the Reynolds Averaged NavierStokes (RANS) equations (Wilcox, 2006). Twenty years passed before the next conceptual leap was made by Prandtl, when he modelled a PDE to express the turbulent kinetic energy, $k$, thereby creating the first one-equation turbulence model. Fundamentally, this allowed the local flow properties to be dependent on antecedent events. Then, van Driest (1956) devised a viscous damping modification to the mixing length model, which has been applied to virtually all algebraic closures since (Wilcox, 2006). A noteworthy example of a zero-equation model includes that devised by Baldwin and Lomax (1978).

Since, the first one-equation model was born, we have witnessed an explosion of competing turbulence closures. In this work, prevalence is given to those, used in our assessment. Thus, we will confine our attention to the main eddy-viscosity models and their significant variants as implemented in the commercial RANS solver, Star-CCM+, version 13.01.011. The fundamental purpose of this class of closures is to predict an eddy-viscosity. Since this was first introduced by Boussinesq, it has since become the underlying hypothesis used to derive the vast majority of turbulence models.

Although the Reynolds Stress turbulence (RST) model boasts as the most physically sound closure strategy (Sarkar and Lakshmanan, 1991), there are problems related to computational stability in its implementation (Parneix et al., 1998). Furthermore, for the present class of problems (ship hydrodynamics), eddy-viscosity models dominate the literature, despite the fact that Rotta (1951) derived the first RST model decades prior to the first practical application of eddy-viscosity-type models. The prevalence of eddy-viscosity models in the field is illustrated at the end of this section.

Broadly speaking, the problem of closure is solved via two main classes of turbulence models. These are the $k-\varepsilon, k-\omega$ models and their variants. Here, $k$ is the turbulent kinetic energy, $\varepsilon$ is the dissipation rate, and $\omega$ is the dissipation frequency. Before we will examine each class sequentially, a qualitative description of a one equation turbulence model is given.

\subsubsection{One equation Spalart-Allmaras turbulence closure}

A popular alternative to two-equation turbulence models are presented in one-equation closures. The most successful of these is the formulation proposed by Spalart and Allmaras (1992). Breaking precedent, this closure uses a diffusivity equation in the place of turbulent kinetic energy to establish an eddy-viscosity. All prior work on models of this type had used the turbulent kinetic energy instead (Baldwin and Timothy, 1990; Johnson and King, 1984). 
The original formulation presented in Spalart and Allmaras (1992) was specifically designed for unstructured codes. Due to its ability to model separated flows and its popularity in the field of aerodynamics (Siemens, 2018), this model is incorporated within the present study.

\subsubsection{The $k-\varepsilon$ model and its variants}

The $k-\varepsilon$ model's foundations were laid by Jones and Launder (1972). It is interesting to note that it the $k-\varepsilon$ model does not owe its popularity to robustness or accuracy, but to the fact that it was the first two equation model applied in practice (Durbin and Pettersson Reif, 2011). That is, even though Kolmogorov's (1942) $k$ - $\omega$ model had been described decades earlier. In Star$\mathrm{CCM}+$, the original coefficients proposed have been replaced with those suggested by Launder and Sharma (1974) in the case of the standard formulation. However, the standard $k-\varepsilon$ model cannot be applied on low Reynolds number (LRN) type cases due to the occurrence of a singularity near solid boundaries. Here, low Reynolds number refers to turbulent $R e_{T}$, which is a function of wall distance $\left(R e_{T}=\sqrt{k} d / v\right.$, where $k$ is the turbulent kinetic energy, $d$ is the distance from the wall to the nearest cell, and $v$ is the viscosity, as opposed to $R e=V L / v$, where $V$ is the speed, and $L$ represents the length of the ship) the near wall treatment of a fully turbulent flow (Durbin, 2017a). Thus, when $y^{+} \sim 1$, models capable of coping with LRN must be used. On the other hand, if $y^{+}>30$, high Reynolds number (HRN) models are required.

Jones and Launder (1972) originally formulated the $k$ - $\varepsilon$ model for HRN-type cases. However, in such a scenario, the use of wall functions is required, which are not compatible with complex flows (Pettersson Reif et al., 2009). Therefore, researchers use either a LRN or realizable versions, discussed below. According to Durbin (1996), two equation turbulence models have a tendency to overpredict turbulent kinetic energy in stagnation points. Realizability refers to the imposition of a suitable constraint to the model to alleviate this shortcoming. Additionally, Star-CCM+ offers the two-layer method, where a PDE is solved for $k$ near the wall, but $\varepsilon$ is algebraically prescribed based on the wall distance (Chen and Patel, 1988). The wall proximity indicator is then used to patch the two solutions onto each other, as suggested by Jongen (1998). On the other hand, the length scale and turbulent viscosity ratio, used to calculate $\varepsilon$ are adopted from Wolfshtein (1969) in the two layer approach.

The realizable, two layer $k-\varepsilon$ model, hereafter referred to as " $k-\varepsilon 2 l$ " for brevity, has been extensively used for practical applications (Cakici et al., 2017; Liu et al., 2018; Ozdemir et al., 2016; Song et al., 2019; Terziev et al., 2018; Tezdogan et al., 2016a, 2016b, to name but a few), making it an ideal candidate for our purposes. However, there are a large number of variants of the $k-\varepsilon$ model put forth by researchers, each claiming superiority. The motivation behind the plethora of alternatives lies with the shortcomings on the model. While its inability to model near-wall effects in the original form can be circumvented easily, the implicitly assumed linear stress-strain relationship poses further problems: linearity may be violated in strongly $3 \mathrm{D}$ boundary layers (Durbin and Pettersson Reif, 2011). This is certainly the case for ship boundary layers, as asserted by Magionesi and Di Mascio, (2016). Despite these shortcomings, the $k-\varepsilon 2 l$ has enjoyed considerable success and widespread implementation for industrial applications. It is therefore interesting to compare the performance of some $k-\varepsilon$ variants available in Star$\mathrm{CCM}+$. Alternatives include proposals by Shih et al. (1995), which cannot be incorporated since it is not offered by the RANS solver.

Abe et al. (1994) proposed a modification of the standard $k-\varepsilon$ model, henceforth referred to as 'AKN' (after the authors of the paper - Abe, Kondoh, Nagano), claiming their variant can 
predict flow reattachment better than the original. As such, their model is of the LRN-type. The authors also altered the coefficients of the original model in their derivation. According to Siemens (2018), the AKN model is a suitable choice for flows over complex geometries, which is undoubtedly representative of a ship underwater shape. For these reasons, this model is selected for the present examination. Additionally, flow reattachment following separation is a crucially important parameter in the prediction of ship resistance, trim and sinkage.

A widely discussed closure in turbulence research is the $k-\varepsilon-v^{2}-f$ model, hereafter referred to as ' $v^{2}-f$ ' (Durbin, 2017a), where $v^{2}$ is the wall-normal stress component and $f$ is the elliptic relaxation parameter. This model solves two additional equations in order to predict the eddyviscosity. Its origins can be traced to work done by Durbin $(1996,1995,1993,1991)$, whereas some recent extensions and modifications include Pettersson Reif et al. (2009), Pettersson Reif, (2006), Laurence et al. (2004), Davidson et al. (2003), and Lien et al. (1998). While this model cannot be classified as a two-equation model for obvious reasons, it retains its larger classification (eddy-viscosity-type model). The benefits of utilising the $v^{2}-f$ model include its ability to handle wall effects, simultaneously accounting for flow nonlocality. Thus, it can be applied directly to LRN-type meshes, without any modifications, or the need to patch regions of different solutions as was the case earlier. Siemens (2018) stated that selecting this model "is known to capture the near-wall turbulence effects more accurately, which is crucial for the accurate prediction of heat transfer, skin friction and flow separation." Thus, it is included in the investigation within the $k-\varepsilon$-class models. The fundamental advantage in implementing the $v^{2}-f$ model is related to its ability to account for anisotropy of turbulence stresses, which are not addressed in the variants examined so far (Gorji et al., 2014).

The final two models incorporated within the present category are elliptic blending closures, henceforth referred to as ' $E B$ '. Both of the variants integrated in this work solve for the turbulent kinetic energy, the dissipation rate, the normalised reduced wall-normal component, and the elliptic blending factor $(\alpha$, rather than $f)$. This is thought to be beneficial due to its inclusion of nonlocal, and pressure echo effects. The latter, observed near impermeable boundaries, are solved for via the method of images. The wall-normal component is defined as the ratio of $v^{2}$ and $k$, and therefore requires the solution of $v^{2}$ in a similar manner to the $v^{2}-f$ model. The concept of elliptic relaxation was first introduced for the RST-class models by Durbin (1993b). As such, it mandated the solution of six equations to provide closure, which was later simplified to a single equation $(\alpha)$. Later, Manceau and Hanjalić (2002) further simplified the $E B$ model rendering it more industry friendly, which is the form utilised by Star$\mathrm{CCM}+$.

The elliptic blending model's conception can be traced to being a consequence of the development of the $v^{2}-f$ model. Up to this point, linear eddy-viscosity models have been the focus. Although, the $v^{2}-f$ has been extended to incorporate nonlinearities, Pettersson Reif (2006) found its linear variant to perform equally well, which is the version employed here. On the other hand, non-linear eddy-viscosity models, specifically of the elliptic blending-type have recently emerged and gained popularity. The main contributors to their development other than Manceau and Hanjalić (2002) include Manceau (2015) and Manceau et al. (2001), however, the conceptual origins of $2^{\text {nd }}$ order closures can be traced to Chou (1945). In the present work, the standard and lag $E B$ (referred to as ' $L a g-E B$ ') are adopted. The former, according to Billard and Laurence (2012), circumvents the $k-\varepsilon 2 l$ models near wall issues, while it exhibits stable performance for practical applications. 
The alterative, the Lag-EB model of Revell et al. (2005), was devised to address a fundamental shortcoming of almost all eddy-viscosity models. Namely, their imposition of a linear relationship between the stress tensor and rate-of-strain tensor, which follows directly from the Boussinesq hypothesis. Revell et al. (2005) implemented a phase lag, or angle between these two parameters to express their misalignment. In Star-CCM+, the implementation of Lag-EB follows the modifications suggested by Billard and Laurence (2012) for stability reasons (Siemens, 2018).

This marks the end of the description of the five $k-\varepsilon$ variants used. The next class of turbulence models replace the dissipation rate, $\varepsilon$, with $\omega$ - the dissipation frequency.

\subsubsection{The $k$ - $\omega$ model and its variants}

The second most widely used strategy to close the governing equations involves the adoption of a $k$ - $\omega$ type turbulence model. Here, only a brief overview of the variants used within this work is given. Wilcox (2006) can be consulted for a comprehensive overview of the subject. It is worth noting that without having prior knowledge of the Kolmogorov (1942) $k-\omega$ model, Saffman (1970) formulated a $k-\omega$ model to close the RANS equations. The latter was applicable down to the wall, immediately showing distinct advantages over the $k$ - $\varepsilon$ model.

The modern version of the $k-\omega$ variant, here referred to as the standard ' $k$ - $\omega$ Wilcox' model, can be traced to Wilcox (1988), although earlier work provided its basis (Saffman and Wilcox, 1974), it was later revised several times by the same author. Most notably, by Wilcox (2008, 2006), the most recent of which is incorporated in Star-CCM+. Siemens (2018) advise that the model coefficients of Wilcox (2008) have not been extensively verified for flows, other than two-dimensional parabolic cases. Therefore, these corrections are included as an optional addon. Since the objective of the present work is to test well-established closures, we have not made use of these.

The $k-\omega$ Wilcox model has faced primarily the criticism of free-stream sensitivity (Cazalbou et al., 1994). This refers to the fact that turbulent statistics must vanish at some distance from an impermeable wall. In the free-stream, their influence on the irrotational region (the region not affected by viscosity) is thought to decay as $\left(y^{+}\right)^{-4}$ (Phillips, 1955) which is not always the case when employing the $k$ - $\omega$ closure. This problem was addressed by Wilcox (2008) and is thought to have been resolved in the latest version of his model. Menter (1994) transformed the transport of $\varepsilon$ from the $k-\varepsilon$ model to resolve the free-stream sensitivity by a variable substitution in the $k$ - $\omega$ Wilcox model. The formulation Menter (1994) arrived at is similar to the standard $k$ - $\omega$ Wilcox model, but contains an additional term expressing the cross-diffusion of $k$ and $\omega$.

In other words, the form derived by Menter (1994), referred to as the ' $k$ - $\omega S S T$ model' (where SST stands for Shear Stress Transport), could potentially provide identical results to the $k-\varepsilon$ model. By incorporating a blending parameter, expressed as a function of wall distance, the $k$ $\omega S S T$ model is thought to preserve the advantages inherited by both main closures used today $(k-\varepsilon$ and $k-\omega)$. This is also one of the models gaining popularity in the field of ship hydrodynamics (Deng et al., 2015; Farkas et al., 2018; Haase et al., 2016; van Wijngaarden, 2005; Wang et al., 2018; Wnęk et al., 2018). As such, it is important that we incorporate it within the present study.

The final aspect of turbulence research considered in this sub-section is associated with the concept of transition. This term is used to describe the change of a boundary layer from laminar 
to turbulent (Saric et al., 2002). This change is of practical importance because a turbulent boundary layer imparts a greater skin friction to the surface from which it is shed, than when in laminar state. Thus, the onset of turbulence, and its possible delay, is of great importance, especially considering that skin friction is one of the main contributing components of ship resistance. According to Durbin (2017b), the primary modes in which transition occurs are:

1. Natural

2. Bypass (as a response to external disturbances)

3. Separation-induced

Transition models are required because most turbulence closures are by definition applicable to the fully turbulent part of a flow (HRN-type models). A related example is the difference and range of applicability of the Blasius (1908) and any other friction lines, for instance, the well-known Schoenherr (1932) line. The $A K N$ model and the standard $k-\varepsilon$ model, for example, contain empirical damping functions, which allow them to exhibit transition properties. However, this is a consequence of solution bifurcation, resulting from the use of damping functions rather than physical insight into the phenomenology of the process itself (Lesieur, 2008). Their use has also been widely criticised (Patel et al., 1984), in part because the only similarity between different empirical damping functions is the presence of exponentials in their derivation (Durbin, 1991; Shih, 1990). For instance, Chien (2008), or Launder and Sharma (1974), to name but a few.

The RANS solver employed in this study, Star-CCM+, offers a variety of methods of modelling transition. The first, and arguably more difficult to implement (the turbulence suppression model) requires prior knowledge of the location of transition. Thus, it is not applicable in many cases. An alternative solution was provided by Abu-Ghannam and Shaw (1980), which is based on the concept of intermittency. This parameter can range from 0 to 1 , describing the time the flow is in a turbulent state (in percentage values). The reason this is not implemented is related to its requirement of nonlocal quantities, which cannot be evaluated at cell centres. A local alternative was suggested by Langtry (2006). Since the formulation was originally incomplete, its adaptation in Star-CCM+ requires user programming and definition of additional functions. Recognising that it is highly unlikely for the average user to accomplish this, we have opted for the simpler ' $\gamma$ ' transition model. Selecting the $\gamma$ transition model activates the solution of an additional intermittency equation $(\gamma)$ and couples its solution with the $k-\omega S S T$ model via the kinetic energy $-k$ (Menter et al., 2015). Non-local effects are handled via an approximation of the three-dimensionality of boundary layer by using the concept of helicity, which is a measure of the cross-flow within a turbulent boundary layer (Müller and Herbst, 2014).

\subsubsection{Potential alternatives}

As has been illustrated thus far, research and development of turbulence models has been rather chaotic, featuring small steps and large conceptual leaps. For instance, while elliptic blendingtype models are thought to be superior, the development of one, and two-equation closures has continued since the former's inception. One way to characterise the field would be via additions of small theoretically and empirically acquired pieces of information to existing models. Indeed, this sort of 'tinkering' is a defining feature of the field of turbulence research (Roache, 2016).

Initially, model constants were calibrated against experiments. When Direct Numerical Simulation (DNS) data became available, the validation procedure shifted. Nowadays, the aim 
is to match DNS data instead of experiments. Regardless, due to the complexity and limitations of the DNS approach, the possible benchmarks are limited to simple two, or three-dimensional cases at best (a classical example is the backward facing step (Darrigol and Turner, 2006)). Thus, the coefficients selected for each model have been calibrated to perform best for these specific cases. It is therefore not possible to determine the precision, accuracy, or even tendency to over or underpredict values of any one turbulence model when applied elsewhere. The matter is worsened by the previously illustrated fact that all turbulence models contain a large degree of empiricism (arrived at by a process of tinkering), compounded by the simple truth the mathematical formulations used do not represent real, physical processes. The degree of empiricism is a reflection of the complexity of the processes occurring in turbulent flows. If analytical solutions were possible, our turbulence models would be exact, and empirical coefficients would not be required (Durbin and Pettersson Reif, 2011). Contrary to expectation, the empiricism is not necessarily a drawback. The problem addressed in this work is our inability to establish a hierarchy in the modelling of turbulence in any meaningful way other than complexity. For this reason, research of the type of Gorji et al. (2014), and El-Behery and Hamed (2011) have emerged attempting to provide specific recommendations with regards to the use of different turbulence closures in a particular context.

Turbulence models described thus far model statistically almost the entirety of the turbulent kinetic energy spectrum. This expresses a concept similar to wave spectra (St Denis and Pierson, 1953), where components of different wavelength coexist simultaneously (turbulent eddies replacing waves in the present context). Richardson (1922) and Kolmogorov (1941) are chiefly attributed with the conceptualisation of the turbulent energy spectrum and its cascade into smaller scales. It is this cascading process that gives rise to nonlinear interactions and thus inhibits its description. Researchers have devised a way to statistically model part of the spectrum via the turbulence models described above, leaving the remainder to be captured in the simulation. This is known as Large (or Very Large) Eddy Simulation (LES or VLES, alternatively, Detached Eddy Simulation - DES). Alongside the twofold increase of the order of magnitude in computational power requirements, Kornev et al. (2019) reported several difficulties when applying the LES technique to ship hydrodynamics problems. The main issue was related to the region used to patch the statistically averaged and resolved solution together. In light of this, the present study will not consider the adoption of LES. Studies, detailing the use of hybrid RANS-LES techniques in marine hydrodynamics include the work of Bhushan et al. (2013), Carrica et al. (2010) and Posa et al. (2019).

Before we proceed with the case-study description, it is worth examining which turbulence models tend to be preferred in marine hydrodynamics. Here, the term 'marine hydrodynamics' is used in reference to research encompassing all aspects of ship and offshore research that includes the simulation of fluid flow using a RANS solver. Additionally, investigations centred on propeller performance are incorporated.

The compiled dataset presents a sample of research work done during the past two decades using RANS solvers in the related field. The sample, collected from the two of biggest academic publishers (ScienceDirect, and Taylor and Francis), is split into categories corresponding to the turbulence model used in each work. No differentiation is made between different subtypes of models (e.g. realizable vs standard $k-\varepsilon$ ). Figure 1 presents the breakdown of the turbulence models among the same categories and provides a graphical representation of their cumulative use over the past roughly two decades. The results shown in Figure 1 
demonstrate that the $k-\omega S S T$ model has gained increasing popularity in the past decade, but virtually all work done is concentrated in two categories. The purpose of this work will then serve to introduce some of the newer turbulence models $\left(v^{2}-f, E B\right.$, and $\left.L a g-E B\right)$ to the field of marine hydrodynamics.

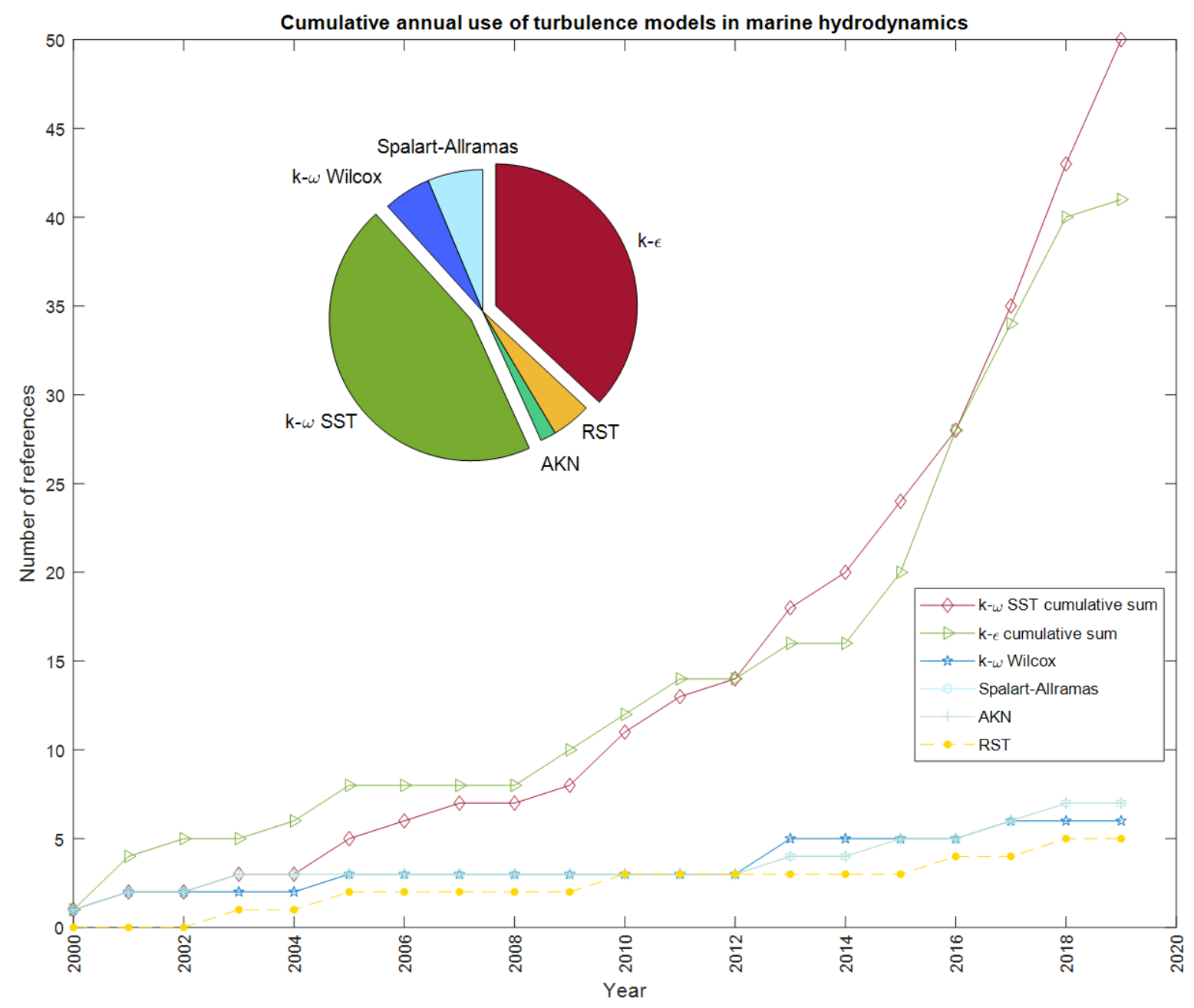

Figure 1. Graphical representation of the use of turbulence models in marine hydrodynamics.

\section{Case-studies}

An integral part of testing the performance of any turbulence model or numerical set-up involves validating results against experimental data. Having established that the present study will focus on shallow water ship hydrodynamics due to the innate complexity of the field, restricts the choices available. In a recent study by Elsherbiny et al. (2019), the behaviour and performance of the well-known benchmark KCS container ship were tested. Although focus was placed on the new Suez Canal in the abovementioned work, a rectangular canal case-study was investigated as well, and is adopted here. Doing so will allow the reduction in cell numbers because simulating the thin layer of water near the intersection of the sloping seabed and sides (as was the case in Tezdogan et al. (2016b)) is bypassed. Thus, the computational mesh can be refined in the vicinity of the ship, and gradually coarsened in every direction without 
compromising the solution. Indeed, doing so is a mainstay in the practice of computational hydrodynamics.

The case-studies examined by Elsherbiny et al. (2019) allow us to vary the speed of the ship and test the turbulence models while keeping all other parameters constant. However, one could argue that in shallow water ship hydrodynamics, a change in the depth can have an effect, comparable to the change in speed. Limiting the case-studies in such a way would prevent the present study's results from being generalised to a wider pool of problems in the field. For this reason, two case-studies adopted from Mucha and el Moctar (2014) are also replicated numerically. In their work, the authors used the same ship as in Elsherbiny et al. (2019), with a different draught ( $T=10 \mathrm{~m}$, rather than $T=10.8 \mathrm{~m}$ full-scale equivalent) and scale factor $(\lambda=40$, rather than $\lambda=75$ in Elsherbiny et al. (2019)). For the purposes of the present research, two ratios of depth and draught have been selected for a single ship speed. This translates into a different Depth Froude number $\left(F_{h}=V / \sqrt{g \times h}\right.$, where $V$ is the speed, $g$ is the gravitational acceleration and $h$ is the depth), although the speed has been maintained constant. $F_{h}$ is commonly thought of as the hydrodynamic equivalent of the Mach number, defining the ratio of ship and wave speeds. In shallow water, waves are nondispersive and can attain a single propagation speed defined by the denominator of the Depth Froude number.

The KCS principle characteristics are summarised in Table 1, while the case-studies are graphically depicted in Figure 2. The conditions, against which the turbulence models are assessed are given in Table 2. All nine turbulence models are tested against each of the four case-studies delineated jointly in Table 1 and Table 2, yielding a total of 36 simulations.

Table 1. Principal characteristics of the KCS

\begin{tabular}{|l|l|l|l|l|}
\hline \multirow{2}{*}{ Quantity } & \multicolumn{2}{|l|}{ Elsherbiny et al. (2019) } & \multicolumn{2}{l|}{ Mucha and el Moctar (2014) } \\
\cline { 2 - 5 } & Full-scale & Model-scale (1:75) & Full-scale & Model-scale $(1: 40)$ \\
\hline$L[\mathrm{~m}]$ & 230 & 3.067 & 230 & 5.75 \\
\hline$B[\mathrm{~m}]$ & 32.2 & 0.429 & 32.2 & 0.805 \\
\hline$T[\mathrm{~m}]$ & 10.8 & 0.144 & 10 & 0.25 \\
\hline$C_{B}[-]$ & 0.651 & 0.651 & 0.64 & 0.64 \\
\hline$S\left[\mathrm{~m}^{2}\right]$ & 9530 & 1.694 & 8992 & 5.62 \\
\hline
\end{tabular}

Table 2. Case-studies description

\begin{tabular}{|c|c|c|c|c|c|c|c|c|}
\hline ID & Reference & $\lambda$ & $h / T$ & $h[\mathrm{~m}]$ & $F_{h}[-]$ & $\operatorname{Re}[-]$ & $V_{\text {model }}[\mathrm{m} / \mathrm{s}]$ & $V_{\text {ship }}[\mathrm{kn}]$ \\
\hline 1 & \multirow{2}{*}{$\begin{array}{c}\text { Elsherbiny et al. } \\
\text { (2019) }\end{array}$} & \multirow{2}{*}{75} & \multirow{2}{*}{2.2} & \multirow{2}{*}{0.32} & 0.303 & $1.8297 \times 10^{6}$ & 0.534 & 9.0 \\
\hline 2 & & & & & 0.57 & $3.4759 \times 10^{6}$ & 1.005 & 16.9 \\
\hline 3 & \multirow{2}{*}{$\begin{array}{l}\text { Mucha and el } \\
\text { Moctar (2014) }\end{array}$} & \multirow{2}{*}{40} & 1.3 & 0.325 & 0.37 & $4.7116 \times 10^{6}$ & \multirow{2}{*}{0.73} & 8.1 \\
\hline 4 & & & 1.6 & 0.4 & 0.41 & $4.7116 \times 10^{6}$ & & 8.1 \\
\hline
\end{tabular}



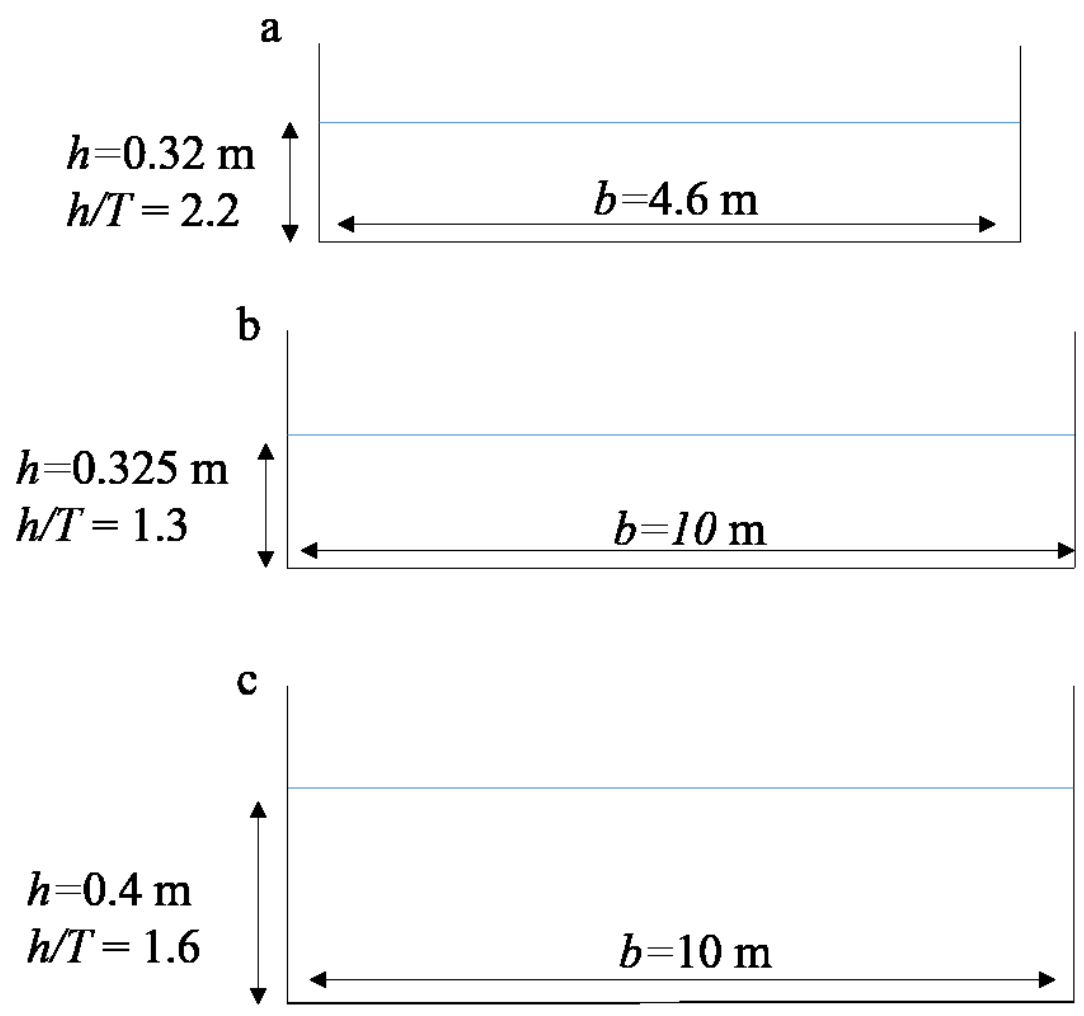

Figure 2. Graphical depiction of the adopted case-studies (not drawn to scale): (a) refers to the study of Elsherbiny et al. (2019), (b) and (c) refer to Mucha and el Moctar (2014).

\section{Numerical implementation}

The RANS solver used in this study, Star-CCM+, employs the Finite Volume Method (FVM) to model the problem at hand by using the integral form of the governing equations and by discretising the computational domain into a finite number of adjoining cells. Within the framework of Star-CCM+, pressure and continuity are linked via a predictor-corrector scheme.

To simulate turbulent properties within the fluid, the closures described in Section 2 are applied. These are summarised in Table 3, where Roman numerals are used to assign each case with a number.

Table 3. Summary of the tested turbulence models.

\begin{tabular}{|c|c|c|}
\hline ID & Class & Identifier \\
\hline $\mathrm{I}$ & \multirow{5}{*}{$k-\varepsilon$} & $A K N$ \\
\hline II & & $v^{2}-f$ \\
\hline III & & $E B$ \\
\hline IV & & $L a g-E B$ \\
\hline $\mathrm{V}$ & & $k-\varepsilon 2 l$ \\
\hline VI & \multirow{3}{*}{$k-\omega$} & $k-\omega \gamma$ \\
\hline VII & & $k-\omega S S T$ \\
\hline VIII & & $k-\omega$ Wilcox \\
\hline IX & One-equation & Spalart-Allmaras \\
\hline
\end{tabular}




\subsection{Physics modelling}

The motion of the fluid is modelled via a flat wave by the Volume of fluid (VOF) method (Hirt and Nichols, 1981). Alternatives include the Level-Set method. However, Starke et al. (2017) showed that some features of the bow wave generated by the ship could not be captured using the Level-Set method. In some deep water cases, the presence of the free surface may be omitted. However, in shallow water flows, the energy imparted on waves due to the disturbance caused by the ship has a higher relative importance than in deep waters. Thus, it is not admissible to neglect the presence of the water surface. A third approach was proposed by Carrica et al. (2007), where a single phase level set method is introduced. This eliminated the need to account for the air filled part of the domain, reducing cell numbers. Unfortunately, it has not been incorporated within the solver used. Therefore, it cannot be applied in the present study.

The phase interface is captured by the RANS solver by assigning a scalar value between 0 and 1 to every cell in the domain. A value of 1 implies the cell is filled with water, conversely, 0 means the cell is in the air-filled part of the domain. A value of 0.5 indicates a cell is half full with air and water, thereby defining the location of the free surface. The adoption of the VOF method requires that both immiscible fluids account for large parts of the domain, while their contact area should be relatively small (Siemens, 2018). To prevent reflections from the outlet, a VOF damping zone, equal to $1.25 \times \mathrm{L}$ is implemented in all cases.

A critical feature of the simulation is the type of convection scheme, which was selected as second order upwind. This choice is made because reducing the order (to $1^{\text {st }}$ order) compromises the accuracy, whereas increasing the order (to $3^{\text {rd }}$ order) can potentially lead to problems related to stability (Siemens, 2018). This is applied to all turbulence models in all case-studies. The segregated flow solver is used solve the Navier-Stokes equations in an uncoupled manner with the aid of a SIMPLE (Semi-Implicit Method for Pressure Linked Equations) algorithm.

To model the motions of the ship, the Dynamic Fluid-Body Interaction (DFBI) module offered by Star-CCM+ is used. Here, the ship is allowed to sink and trim only, which occur as a result of pressure (normal) and shear (tangential) forces acting on the hull. To avoid a large initial shock, resulting when the simulation is initiated, the ship is constrained during the first 5 seconds in all simulations. Implementing this constraint on the motions of the ship reduces the transient oscillatory motion typically observed in the early stages of the simulation. Since the type of problem is pseudo-steady, it is desirable to reduce the time taken to achieve a steady state. The time taken for the solver to complete a single iteration is also reduced during these first 5 seconds, since an update to the position of the ship is not required at the end of the inner loop, which is set to comprise of 10 iterations. It is important to note that the free surface is updated at every iteration, while sinkage, trim, and resistance are recorded and updated once the inner iterations loop has competed.

\subsubsection{Time step selection}

At the end of the inner loop of iterations, the time step must be updated. This is selected in all cases as $\Delta t=0.0035 \mathrm{~L} / \mathrm{V}$, following Terziev et al., (2018), and Tezdogan et al. (2016b), where the efficacy of this method of setting $\Delta t$ has been demonstrated. This value is calculated for each case-study, but is not changed across turbulence models. An advantage of using this method of setting the time step is that we do not need to change the mesh when the speed is 
altered. Thus, for the four case-studies, only 3 meshes are required. Cases 1 and 2 can be used with the same computational mesh (whose properties are examined in the following subsection), while the time step is altered according to the formula above. Case-study 4 requires a slight increase in cell numbers due to the different depth, when compare to case 3 (see Section 4.1.3, Table 4). All other characteristics, including the time step for cases 3 and 4 is the same because the speed is not changed. Alternative approaches to setting the time step include the ITTC's (2011) $\Delta t=0.005 \sim 0.01 \mathrm{~L} / \mathrm{V}$. Throughout all examined cases, the temporal discretisation is set as first order, and the solution is allowed to develop for a minimum of 150 seconds of physical time to ensure convergence of the results.

\subsubsection{Computational domain}

The computational domain has been structured according to the recommendations of ITTC (2011) and Siemens (2018). Namely, the inlet boundary in resistance computations should be located between one and two ship lengths from the ship bow, while the outlet - between two and three ship lengths downstream. In this work, the velocity inlet is placed 1.5 ship lengths upstream from the forward perpendicular, while the pressure outlet -2.5 ship lengths downstream from the aft perpendicular. The domain width and depth are dictated by the casestudies, and are summarised in Table 2. The side boundary is set as a wall, while the domain bottom is a velocity inlet. The latter choice is made because the cells in the domain are linked to the ship's centre of gravity $(\mathrm{CoG})$, which is set as the origin of the local coordinate system. As the CoG translates and rotates, some cells cross the domain bottom. To ensure that the same amount of water flows past the ship, the domain bottom is allowed to introduce the lost amount of water back into the domain. This is done adding the same VOF flat wave speed to the domain bottom in the negative $x$ - direction. In other words, the bottom acts as a reflective boundary, without developing a boundary layer as a result of the relative motion between it and the fluid, while simultaneously ensuring the $h / T$ condition is maintained.

To alleviate the computational cost, a symmetry boundary is imposed, coincident with the ship and canal centreline. Finally, the domain top is located 1.25 ship lengths from the undisturbed water level, where a velocity inlet condition is imposed. The domain is graphically shown in Figure 3.

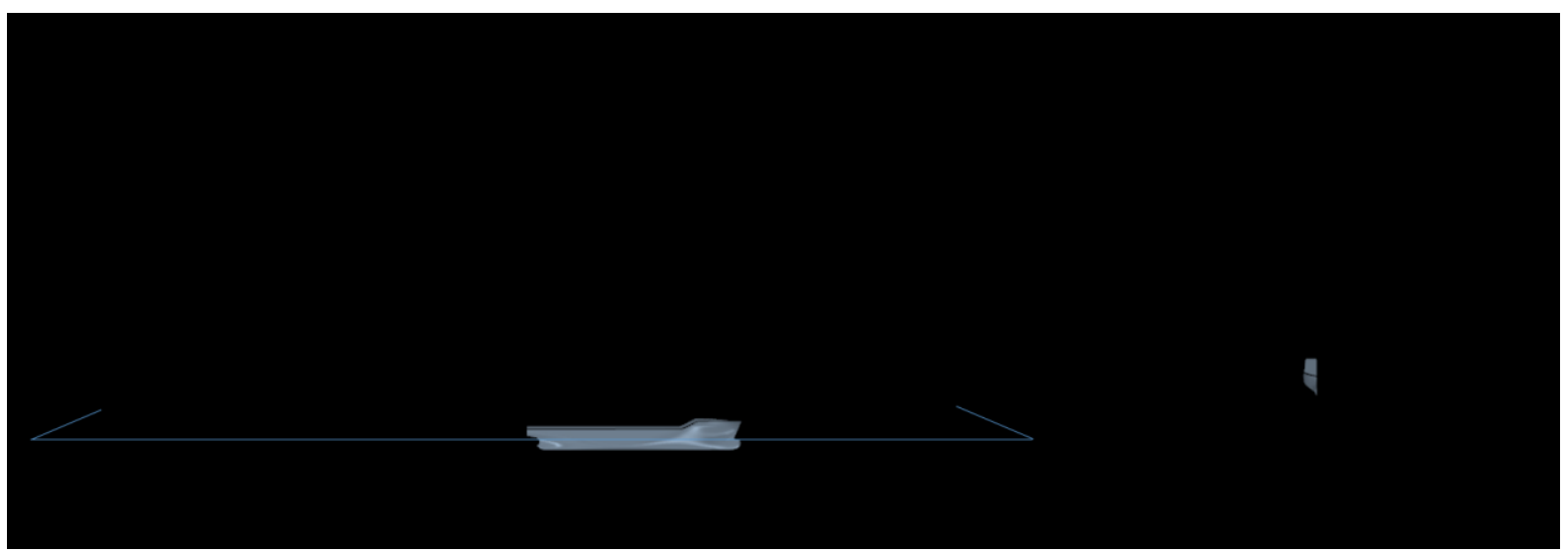

Figure 3. Boundary conditions and dimensions for all computational domains simulated in this study. 


\subsubsection{Mesh generation}

The mesh was generated within the facilities of Star-CCM+, which allow the user to make full use of the software's automatic operations. The static, region-based mesh comprises mainly of hexahedral cells with minimal skewness, generated via the trimmed cell mesher. According to the findings of Jones and Clarke (2010), tetrahedral cells can compromise the accuracy of the solution. Concentric local volumetric refinements are imposed in the vicinity of the hull, ensuring the accurate representation of flow phenomena. Furthermore, the location where the free surface is expected to deform has been systematically refined.

The prism layer mesher is used to capture the ship boundary layer by creating orthogonal prismatic cells. Resolving near-wall flow accurately is of critical importance, which is why the $y^{+}$values are monitored, ensuring the average values remain below 1 in all cases (Eça et al., 2018). The distribution of $y^{+}$values along the wetted part of the hull are shown in Figure 4 for cases 1 and 3.
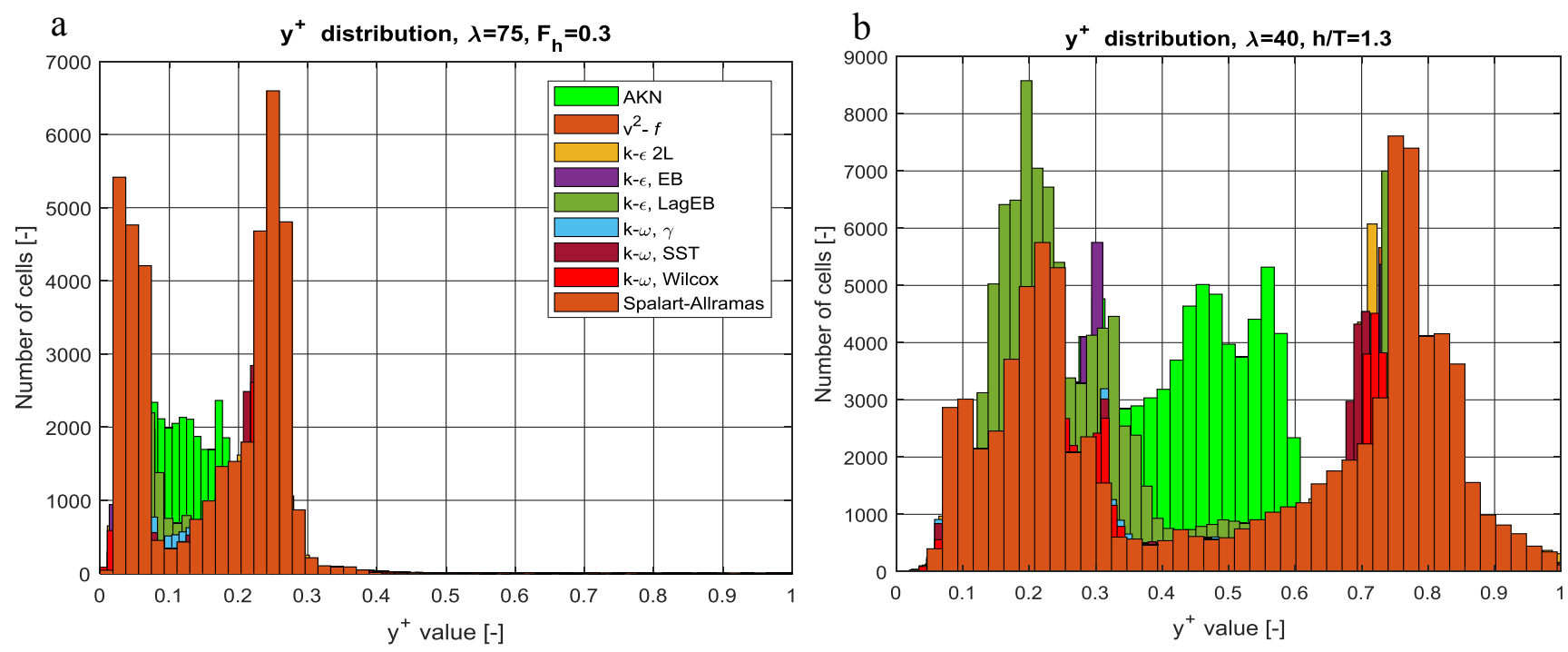

Figure 4. Sample $y^{+}$value distribution along the wetted hull: (a) refers to the study of Elsherbiny et al. (2019), (b) refers to Mucha and el Moctar (2014) - case 2.

While the vast majority of turbulence models predict the same $y^{+}$values, the $A K N$ model deviates significantly. In the next section, we will show that such behaviour leads to significantly compromised accuracy. For case 1 (of Elsherbiny et al. (2019)) the large scale factor $(\lambda=75)$ allows an increased resolution to be imposed without the need to resort to an excessively large number of cells. The second case is not as straightforward, which is why the $y^{+}$values are allowed to reach 0.9 , where the average of in all cases is smaller than 1 , despite the two-fold increase in cell numbers (Table 4). The resulting computational mesh, mirrored using the central symmetry for illustration purposes, is shown in Figure 5, where the local volumetric refinements are clearly visible, including the Kelvin wedge. The properties of the computational domain for all case-studies are shown in Table 4.

Strictly speaking, the imposition of a symmetry plane has the potential of invalidating the results presented herein. However, Mucha and el Moctar (2014) demonstrated a negligible difference resulting from the presence of a symmetry plane. For this reason, it is deemed safe to proceed directly with the computational mesh, as described previously. Finally, to enable a reliable comparison of the results, free of unregistered unknowns, all parameters described in this section have been kept identical across the case-studies. 


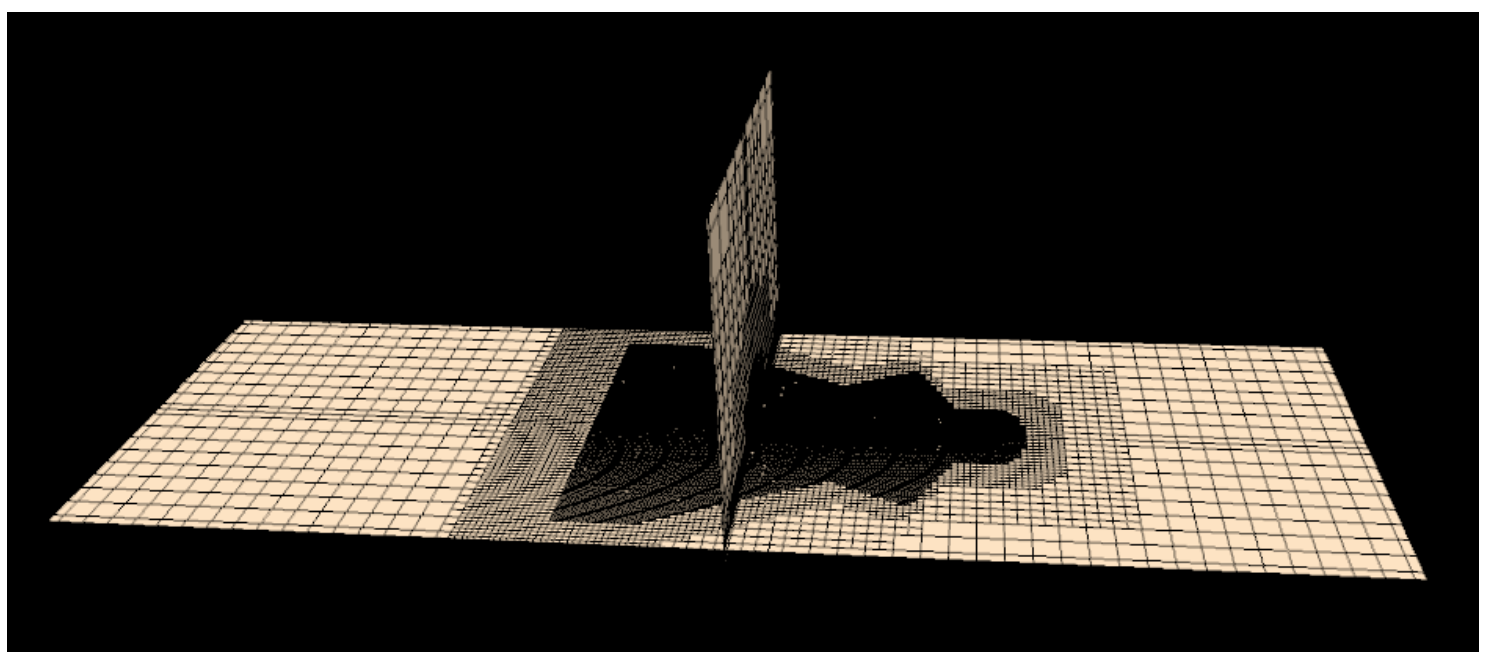

Figure 5. 3-D view of the computational mesh. Depicted: case 1 (mirrored using the central symmetry plane).

Table 4. Computational domain cell properties.

\begin{tabular}{|l|l|l|l|}
\hline Case-study number & Number of cells & Number of faces & Number of vertices \\
\hline 1 and 2 & 1446076 & 4281940 & 1491537 \\
\hline 3 & 2995685 & 8868880 & 3087140 \\
\hline 4 & 3032015 & 8984576 & 3125549 \\
\hline
\end{tabular}

\section{Results and discussion}

In this section, the results are presented and compared, both against each other and against the relevant experiments. The first sub-section is dedicated to the numerical uncertainties and error estimations against experiments. Presenting these first will later allow a fuller discussion of the results.

\subsection{Validation and verification}

\subsubsection{Validation against experimental data}

The first step taken in this section is to present the results in tabular form alongside the experimentally obtained values (EFD - Experimental Fluid Dynamics) and calculated error (E). This step is used to validate the solution. The error is defined as $E=(E F D-C F D) / E F D \times 100$, and is shown in Table 5. The values, highlighted in red were not used in the computation of averages shown in the same table. This is done in an attempt to allow the global average (shown in bold) of the error to represent the values in a meaningful way. If the error values for trim were taken into account, the global average error would be much higher.

The first conclusion drawn from the errors shown in Table 5 suggest that resistance can be predicted by almost all turbulence models effectively. This is true especially considering the experimental force measurement uncertainty, which is up to $2.20 \%$ in Elsherbiny et al. (2019). As alluded to previously, sinkage and trim present an additional layer of complexity. The numerical set-up has not predicted values within acceptable margins for one row (case 3), in the case of sinkage and three (cases 1, 3, and 4) in the case of trim (discussed further in the following sections). This is the case for two reasons, which include numerical and experimental uncertainty. 
Table 5. Numerical results and error calculations

\begin{tabular}{|c|c|c|c|c|c|c|c|c|c|c|c|c|c|c|c|}
\hline \multicolumn{4}{|c|}{ Description } & \multirow{2}{*}{ ID } & \multirow{2}{*}{ Type } & \multirow{2}{*}{$A K N$} & \multirow{2}{*}{$v^{2}-f$} & \multirow{2}{*}{$E B$} & \multirow{2}{*}{ Lag-EB } & \multirow{2}{*}{$k-\varepsilon 2 l$} & \multirow{2}{*}{$k-\omega \gamma$} & \multirow{2}{*}{$k-\omega S S T$} & \multirow{2}{*}{$k-\omega$ Wilcox } & \multirow{2}{*}{$S P-A l l$} & \multirow{2}{*}{ Average } \\
\hline \multirow{13}{*}{ 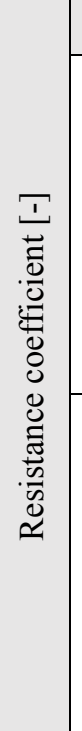 } & $\lambda$ & $F_{h}$ & $h / T$ & & & & & & & & & & & & \\
\hline & \multirow{6}{*}{75} & \multirow{3}{*}{0.303} & \multirow{6}{*}{2.2} & \multirow{3}{*}{1} & EFD & \multicolumn{9}{|c|}{$5.775 \times 10^{-3}$} & - \\
\hline & & & & & CFD & $4.401 \times 10^{-3}$ & $5.559 \times 10^{-3}$ & $5.559 \times 10^{-3}$ & $5.614 \times 10^{-3}$ & $5.439 \times 10^{-3}$ & $5.298 \times 10^{-3}$ & $5.340 \times 10^{-3}$ & $5.505 \times 10^{-3}$ & $5.596 \times 10^{-3}$ & $5.489 \times 10^{-3}$ \\
\hline & & & & & $E$ & $23.79 \%$ & $3.74 \%$ & $3.75 \%$ & $2.79 \%$ & $5.83 \%$ & $8.26 \%$ & $7.53 \%$ & $4.67 \%$ & $3.10 \%$ & $4.96 \%$ \\
\hline & & \multirow{3}{*}{0.570} & & \multirow{3}{*}{2} & EFD & \multicolumn{9}{|c|}{$5.224 \times 10^{-3}$} & - \\
\hline & & & & & CFD & $4.087 \times 10^{-3}$ & $5.212 \times 10^{-3}$ & $5.199 \times 10^{-3}$ & $5.247 \times 10^{-3}$ & $5.096 \times 10^{-3}$ & $5.034 \times 10^{-3}$ & $5.014 \times 10^{-3}$ & $5.138 \times 10^{-3}$ & $5.239 \times 10^{-3}$ & $5.147 \times 10^{-3}$ \\
\hline & & & & & $E$ & $21.77 \%$ & $0.24 \%$ & $0.49 \%$ & $-0.44 \%$ & $2.46 \%$ & $3.64 \%$ & $4.02 \%$ & $1.65 \%$ & $-0.27 \%$ & $1.47 \%$ \\
\hline & \multirow{6}{*}{40} & \multirow{3}{*}{0.370} & \multirow{3}{*}{1.3} & \multirow{3}{*}{3} & EFD & \multicolumn{9}{|c|}{$2.784 \times 10^{-3}$} & - \\
\hline & & & & & CFD & $1.120 \times 10^{-3}$ & $2.802 \times 10^{-3}$ & $2.668 \times 10^{-3}$ & $2.869 \times 10^{-3}$ & $2.851 \times 10^{-3}$ & $2.587 \times 10^{-3}$ & $2.559 \times 10^{-3}$ & $2.661 \times 10^{-3}$ & $2.842 \times 10^{-3}$ & $2.730 \times 10^{-3}$ \\
\hline & & & & & $E$ & $59.76 \%$ & $-0.64 \%$ & $4.15 \%$ & $-3.06 \%$ & $-2.42 \%$ & $7.07 \%$ & $8.09 \%$ & $4.42 \%$ & $-2.07 \%$ & $1.94 \%$ \\
\hline & & & & & EFD & & & & & $4.74 \times 10^{-3}$ & & & & & - \\
\hline & & 0.410 & 1.6 & 4 & CFD & $1.80 \times 10^{-3}$ & $5.02 \times 10^{-3}$ & $4.67 \times 10^{-3}$ & $4.99 \times 10_{-3}$ & $4.97 \times 10^{-3}$ & $4.49 \times 10^{-3}$ & $4.45 \times 10^{-3}$ & $4.66 \times 10^{-3}$ & $4.98 \times 10^{-3}$ & $4.781 \times 10^{-3}$ \\
\hline & & & & & $E$ & $62.02 \%$ & $-5.86 \%$ & $1.50 \%$ & $-5.27 \%$ & $-4.79 \%$ & $5.28 \%$ & $6.24 \%$ & $1.89 \%$ & $-5.01 \%$ & $-0.75 \%$ \\
\hline & & & & & EFD & & & & & $-2.09 \times 10^{-3}$ & & & & & - \\
\hline & & 0.303 & & 1 & CFD & $-2.09 \times 10^{-3}$ & $-2.00 \times 10^{-3}$ & $-2.00 \times 10^{-3}$ & $-2.01 \times 10^{-3}$ & $-2.01 \times 10^{-3}$ & $-2.02 \times 10^{-3}$ & $-2.03 \times 10^{-3}$ & $-2.02 \times 10^{-3}$ & $-2.01 \times 10^{-3}$ & $-2.015 \times 10^{-3}$ \\
\hline & & & 00 & & $E$ & $-0.07 \%$ & $4.10 \%$ & $4.18 \%$ & $3.58 \%$ & $3.54 \%$ & $3.23 \%$ & $2.97 \%$ & $3.09 \%$ & $3.65 \%$ & $3.14 \%$ \\
\hline & 15 & & 2.2 & & EFD & & & & & $-8.85 \times 10^{-3}$ & & & & & - \\
\hline 7 & & 0.570 & & 2 & CFD & $-8.48 \times 10^{-3}$ & $-8.18 \times 10^{-3}$ & $-8.20 \times 10^{-3}$ & $-8.22 \times 10^{-3}$ & $-8.19 \times 10^{-3}$ & $-8.25 \times 10^{-3}$ & $-8.28 \times 10^{-3}$ & $-8.25 \times 10^{-3}$ & $-8.18 \times 10^{-3}$ & $-8.221 \times 10^{-3}$ \\
\hline$\frac{\Xi}{0}$ & & & & & $E$ & $4.15 \%$ & $7.52 \%$ & $7.29 \%$ & $7.09 \%$ & $7.41 \%$ & $6.76 \%$ & $6.45 \%$ & $6.78 \%$ & $7.57 \%$ & $7.11 \%$ \\
\hline 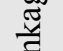 & & & & & EFD & & & & & $-6.16 \times 10-3$ & & & & & - \\
\hline क & & 0.37 & 1.3 & 3 & CFD & $-7.73 \times 10^{-3}$ & $-7.60 \times 10^{-3}$ & $-7.54 \times 10^{-3}$ & $-7.56 \times 10^{-3}$ & $-7.69 \times 10^{-3}$ & $-7.61 \times 10^{-3}$ & $-7.61 \times 10^{-3}$ & $-7.60 \times 10^{-3}$ & $-7.51 \times 10^{-3}$ & $-7.591 \times 10^{-3}$ \\
\hline & H & & & & $E$ & $-25.46 \%$ & $-23.35 \%$ & $-22.47 \%$ & $-22.72 \%$ & $-24.83 \%$ & $-23.59 \%$ & $-23.59 \%$ & $-23.34 \%$ & $-22.00 \%$ & $-23.24 \%$ \\
\hline & 40 & & & & EFD & & & & & $-6.16 \times 10^{-3}$ & & & & & - \\
\hline & & 0.41 & 1.6 & 4 & CFD & $-6.01 \times 10^{-3}$ & $-5.83 \times 10^{-3}$ & $-5.85 \times 10^{-3}$ & $-5.83 \times 10^{-3}$ & $-5.88 \times 10^{-3}$ & $-5.86 \times 10^{-3}$ & $-5.89 \times 10^{-3}$ & $-5.88 \times 10^{-3}$ & $-5.77 \times 10^{-3}$ & $-5.848 \times 10^{-3}$ \\
\hline & & & & & $E$ & $2.49 \%$ & $5.28 \%$ & $5.11 \%$ & $5.37 \%$ & $4.58 \%$ & $4.85 \%$ & $4.37 \%$ & $4.60 \%$ & $6.38 \%$ & $5.07 \%$ \\
\hline 묻 & 75 & 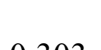 & 00 & 1 & EFD & & & & & $-2.2 \times 10^{-2}$ & & & & & - \\
\hline 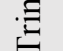 & 15 & 0.303 & 2.2 & 1 & CFD & $-3.79 \times 10^{-2}$ & $-3.56 \times 10^{-2}$ & $-3.52 \times 10^{-2}$ & $-3.58 \times 10^{-2}$ & $-3.55 \times 10^{-2}$ & $-3.58 \times 10^{-2}$ & $-3.54 \times 10^{-2}$ & $-3.59 \times 10^{-2}$ & $-3.64 \times 10^{-2}$ & $-3.568 \times 10^{-2}$ \\
\hline
\end{tabular}




\begin{tabular}{|c|c|c|c|c|c|c|c|c|c|c|c|c|c|c|}
\hline & & & & $E$ & $-69.15 \%$ & $-58.78 \%$ & $-56.92 \%$ & $-59.82 \%$ & $-58.38 \%$ & $-59.67 \%$ & $-57.84 \%$ & $-60.35 \%$ & $-62.29 \%$ & \multirow{3}{*}{$\begin{array}{c}-60.36 \% \\
- \\
-1.005 \times 10^{-1}\end{array}$} \\
\hline & \multirow{3}{*}{0.570} & & \multirow{3}{*}{2} & EFD & & \\
\hline & & & & CFD & \multicolumn{9}{|c|}{$\begin{array}{l}-1.03 \times 10^{-1} \\
-995 \times 10^{-2} \\
-102 \times 10^{-1}\end{array}$} & \\
\hline & & & & $E$ & $-4.49 \%$ & $3.22 \%$ & $5.20 \%$ & $3.12 \%$ & $3.21 \%$ & $1.27 \%$ & $1.82 \%$ & $0.67 \%$ & $-0.62 \%$ & $1.49 \%$ \\
\hline \multirow{6}{*}{40} & \multirow{3}{*}{0.37} & \multirow{3}{*}{1.3} & \multirow{3}{*}{3} & EFD & \multicolumn{9}{|c|}{$-2.24 \times 10^{-2}$} & - \\
\hline & & & & CFD & $3.97 \times 10^{-4}$ & $-5.39 \times 10^{-3}$ & $-6.01 \times 10^{-3}$ & $-5.67 \times 10^{-3}$ & $-4.81 \times 10^{-3}$ & $-5.44 \times 10^{-3}$ & $-4.97 \times 10^{-3}$ & $-4.99 \times 10^{-3}$ & $-6.23 \times 10^{-3}$ & $-5.438 \times 10^{-3}$ \\
\hline & & & & $E$ & $101.77 \%$ & $75.93 \%$ & $73.16 \%$ & $74.69 \%$ & $78.49 \%$ & $75.70 \%$ & $77.77 \%$ & $77.73 \%$ & $72.19 \%$ & $75.71 \%$ \\
\hline & \multirow{3}{*}{0.41} & \multirow{3}{*}{1.6} & \multirow{3}{*}{4} & EFD & \multicolumn{9}{|c|}{$-2.24 \times 10^{-2}$} & - \\
\hline & & & & CFD & $-9.10 \times 10^{-4}$ & $-5.37 \times 10^{-3}$ & $-5.48 \times 10^{-3}$ & $-4.86 \times 10^{-3}$ & $-4.98 \times 10^{-3}$ & $-5.42 \times 10^{-3}$ & $-5.03 \times 10^{-3}$ & $-4.88 \times 10^{-3}$ & $-6.54 \times 10^{-3}$ & $-5.319 \times 10^{-3}$ \\
\hline & & & & $E$ & $95.93 \%$ & $76.03 \%$ & $75.53 \%$ & $78.27 \%$ & $77.74 \%$ & $75.78 \%$ & $77.55 \%$ & $78.19 \%$ & $70.79 \%$ & $76.24 \%$ \\
\hline \multicolumn{5}{|c|}{ Average } & $24.57 \%$ & $2.20 \%$ & $3.96 \%$ & $1.65 \%$ & $2.48 \%$ & $5.05 \%$ & $5.19 \%$ & $3.47 \%$ & $1.59 \%$ & $3.13 \%$ \\
\hline
\end{tabular}


Particularly in the case of trim for case-studies 3 and 4, no turbulence model has adequately predicted the experimentally measured results. This can stem from a variety of sources. One interesting observation is that in Mucha and el Moctar (2014) the reported Longitudinal Centre of Gravity (LCG) is different from what is typically reported elsewhere. In the numerical work done here, the LCG is located as prescribed in the relevant reference. However, this serves to highlight a problem in the results: the two pieces of research found a disagreement in a metric as fundamental as the LCG. In the presence of such uncertainties, it is imperative that an interfacility test is conducted and assessed via a Youden (1972) style technique.

\subsubsection{Numerical verification}

The numerical verification adopted herein follows widely used procedures in the ship hydrodynamics community (Xing and Stern, 2010). Specifically, the Richardson Extrapolation (RE) procedure (Richardson, 1911). This consists of expressing the error as an expanded power series with integer powers of grid spacing $(\Delta x)$ or time step $(\Delta t)$ as a finite sum. If one assumes the solutions lie within the asymptotic range, it is admissible to take only the first term into account, leading to the so-called grid triplet study. Following Xing and Stern (2010), the Grid Convergence Index (GCI) is used, devised by Roache (1998). This method can be used to establish the uncertainty due to grid spacing and time step errors, as demonstrated in Terziev et al. (2018), Tezdogan et al. (2016b). This step is a crucially important part of any numerical solution, and its omission in computational science and engineering is not admissible (Roache et al., 1986). On the other hand, the iterative errors are calculated as being virtually zero in all cases following the procedure of Roy and Blottner (2006).

The GCI procedure begins by calculating the convergence ratio $\left(R_{k}\right)$, defined as the ratio between $\varepsilon_{k 21}=\left(\varphi_{k 2}-\varphi_{k 1}\right)$ and $\varepsilon_{k 32}=\left(\varphi_{k 3}-\varphi_{k 2}\right)$. Here, $\varphi_{k i}$ refers to the solution obtained via the $\mathrm{k}^{\text {th }}$ input parameter using the $i^{\text {th }}$ solution. The solutions $(i)$ are obtained by systematically coarsening the $\mathrm{k}^{\text {th }}$ parameter by a factor of $\sqrt{2}$ (also known as the refinement ratio $-r_{k}$ ), as recommended by ITTC (2008). Thus, in the case of grid dependence, the base size is magnified by the above factor, whereas in the case of time dependence, the time step is lessened by $r_{k}$. This procedure yields a total of four extra solutions, used to define four possible scenario for $R_{k}$ for time and grid dependence (Stern et al., 2006):

1. Monotonic convergence, if $0>R_{k}>1$

2. Oscillatory convergence, if $R_{k}<1$ and $\left|R_{k}\right|<1$

3. Monotonic divergence, if $R_{k}>1$

4. Neither error nor uncertainty can be evaluated

Once the type of convergence is known, the order of accuracy (Celik et al., 2008), $p_{k}$ is calculated as shown in Eq. (1). A monotonic convergence is assumed in all equations presented herein.

$p_{k}=\frac{\ln \left(\varepsilon_{k 23} / \varepsilon_{k 21}\right)}{\ln \left(r_{k}\right)}$

The next step is to find the extrapolated solution - Eq. (2), relative error - Eq. (3), and extrapolated error - Eq. (4):

$\varphi_{\text {ext }}^{21}=\left(r_{21}^{p} \times \varphi_{1}-\varphi_{2}\right) /\left(r_{21}^{p}-1\right)$

$e_{a}^{21}=\left|\left(\varphi_{1}-\varphi_{2}\right) / \varphi_{1}\right|$ 
$e_{\text {ext }}^{21}=\left|\left(\varphi_{\text {ext }}^{12}-\varphi_{1}\right) / \varphi_{\text {ext }}^{12}\right|$

Finally, the grid convergence index can be calculated as shown in Eq. (5):

$G C I_{\text {fine }}^{21}=1.25 e_{a}^{21} /\left(r_{21}^{p}-1\right)$

To obtain the solutions in the grid convergence study, the smallest time step is used while coarsening the grid. On the other hand, to obtain the time step convergence study results, the finest grid is used while lessening the time step. Throughout the numerical verification procedure, surface mesh characteristics were kept constant to maintain the accurate representation of the ship geometry. The convergence studies are summarised in Table 6 . The results suggest that simulations are subject to greater uncertainty stemming from the grid, rather than the time step. Although a GCI of $12.47 \%$ (for sinkage) may seem high, Elsherbiny et al. (2019) report a 90\% uncertainty for sinkage and trim. In other words, the simulations exhibit uncertainty, with an order of magnitude smaller than the experiment. The reason why this is the case is related to experimental measurement equipment. The displacements used to calculate sinkage and trim are too small to be reliably measured. Even in the case of resistance, the numerical uncertainty $(1.94 \%$ and $0.01 \%)$ is smaller than the experimental uncertainty $2.2 \%$. This also explains the observed errors in Table 5 . While we have predicted the resistance reasonably well in all cases, sinkage and trim are harder to capture, both experimentally and numerically.

Table 6. Convergence study for sinkage, trim, and resistance for case $1, \lambda=75, h / T=2.2$, $F_{h}=0.303$, EB model.

\begin{tabular}{|l|l|l|l|l|l|l|}
\hline \multirow{2}{*}{ Parameter } & \multicolumn{3}{|c|}{ Grid dependence } & \multicolumn{3}{|c|}{ Time dependence } \\
\cline { 2 - 7 } & Sinkage $[\mathrm{m}]$ & Trim $\left[^{\circ}\right]$ & Resistance $[\mathrm{N}]$ & Sinkage $[\mathrm{m}]$ & Trim [ $\left.{ }^{\circ}\right]$ & Resistance $[\mathrm{N}]$ \\
\hline$r$ & $\sqrt{2}$ & $\sqrt{2}$ & $\sqrt{2}$ & $\sqrt{2}$ & $\sqrt{2}$ & $\sqrt{2}$ \\
\hline$\varphi_{1}$ & $-2.002 \times 10^{-3}$ & $-3.516 \times 10^{-2}$ & 1.252 & $-2.002 \times 10^{-3}$ & $-3.516 \times 10^{-2}$ & 1.252 \\
\hline$\varphi_{2}$ & $-2.181 \times 10^{-3}$ & $-3.402 \times 10^{-2}$ & 1.269 & $-2.000 \times 10^{-3}$ & $-3.510 \times 10^{-2}$ & 1.252 \\
\hline$\varphi_{3}$ & $-2.655 \times 10^{-3}$ & $-3.209 \times 10^{-2}$ & 1.316 & $-1.946 \times 10^{-3}$ & $-3.498 \times 10^{-2}$ & 1.251 \\
\hline$R$ & 0.380 & 0.591 & 0.360 & 0.367 & 0.575 & 0.111 \\
\hline$p$ & 2.792 & 1.519 & 2.900 & 9.536 & 1.599 & 6.334 \\
\hline$\varphi_{\text {ext }}^{12}$ & $-1.822 \times 10^{-3}$ & $-3.630 \times 10^{-2}$ & 1.235 & $-2.004 \times 10^{-3}$ & $-3.523 \times 10^{-2}$ & 1.252 \\
\hline$e_{a}^{21}$ & $8.99 \%$ & $3.25 \%$ & $1.37 \%$ & $0.10 \%$ & $0.18 \%$ & $0.01 \%$ \\
\hline$e_{\text {ext }}^{21}$ & $9.88 \%$ & $3.14 \%$ & $1.39 \%$ & $0.10 \%$ & $0.18 \%$ & $0.00 \%$ \\
\hline$G_{\text {GCI }}^{21}$ fine & $12.47 \%$ & $7.56 \%$ & $1.94 \%$ & $0.19 \%$ & $0.38 \%$ & $0.01 \%$ \\
\hline
\end{tabular}

Finally, the modelling errors induced by a change in turbulence models are known to differ depending on the closure selected (Pereira et al., 2017). Therefore, the results presented in Table 6 are not uniform across all case-studies. However, having validated the solution against experimental data, it is not though necessary to verify the numerical solution for each turbulence model. The number of additional solutions required per case study (four) implies an unreasonably high number (140) of additional simulations necessary to bind the numerical 
error completely, as would be desirable. In consequence, due to the extreme computational expense associated with the procedure, this is left as a piece of future work.

\subsection{Results comparison}

Having established that resistance is predicted reasonably well by all turbulence models (with the exception of $A K N$ ), it is worthwhile examining its constituent components in more detail. In CFD assessments the resistance of a body subject to fluid flow is defined as the sum of tangential (shear) and normal (pressure) components. Each of these is non-dimensionalised by division by $0.5 \rho V^{2} S$, where $\rho=997.561 \mathrm{~kg} / \mathrm{m}^{3}$ is the water density, and $S$ is the wetted surface area $\left(\mathrm{C}_{\mathrm{F}}\right.$ and $\mathrm{C}_{\mathrm{P}}$, respectively). Figure 6-Figure 9 show the distribution of resistance among the two categories (shear and pressure) as well the experimental values and error calculated for each turbulence model. In the figures, the errors calculated by the $A K N$ model have not been included in order to preserve the $y$-scale of the error within reasonable values for the remaining turbulence closures.

\subsubsection{Pressure resistance}

The results (Figures Figure 6-Figure 9) suggest that all turbulence models predict the pressure component of resistance well, with little disagreement between the different approaches to closing the governing equations. This may seem a trivial observation, since the pressure resistance is not strongly coupled with viscous flow behaviour near impermeable walls. This has been widely used as a justification for the coupling of RANS and potential flow solvers (Tahara and Stern, 1994). However, this observation has wider implications: it suggests the wave resistance, which is part of pressure resistance, is computed with good accuracy regardless of the turbulence model. Wave resistance has been a particularly important and difficult metric to accurately capture in the past for several reasons. These include the complexity of the flow surrounding a ship. In some cases, the deep water wave resistance problem is thought to be addressed reasonably well by potential flow. However, the vast majority of theories break down for shallow waters. A good example illustrating this is Michell's (1898) integral. As shown by Tuck and Lazauskas (2008), Beck (1977), Beck et al., (1975), Tuck (1967, 1966), Michell's (1898) integral can be extended to a large variety of shallow water problems, including deep water wave resistance, but not to the prediction of shallow water wave resistance. 


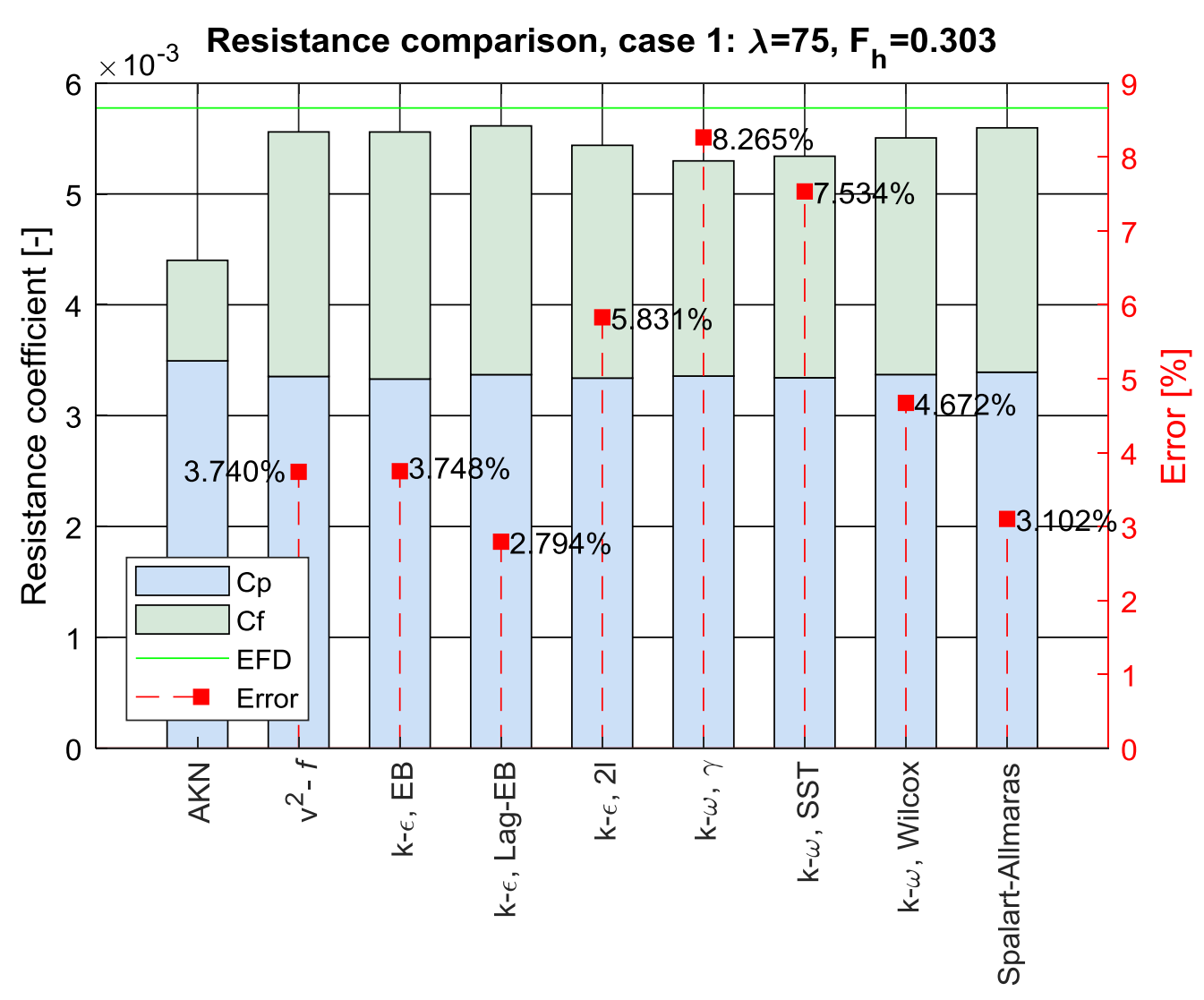

Figure 6. Resistance coefficients comparison for case 1

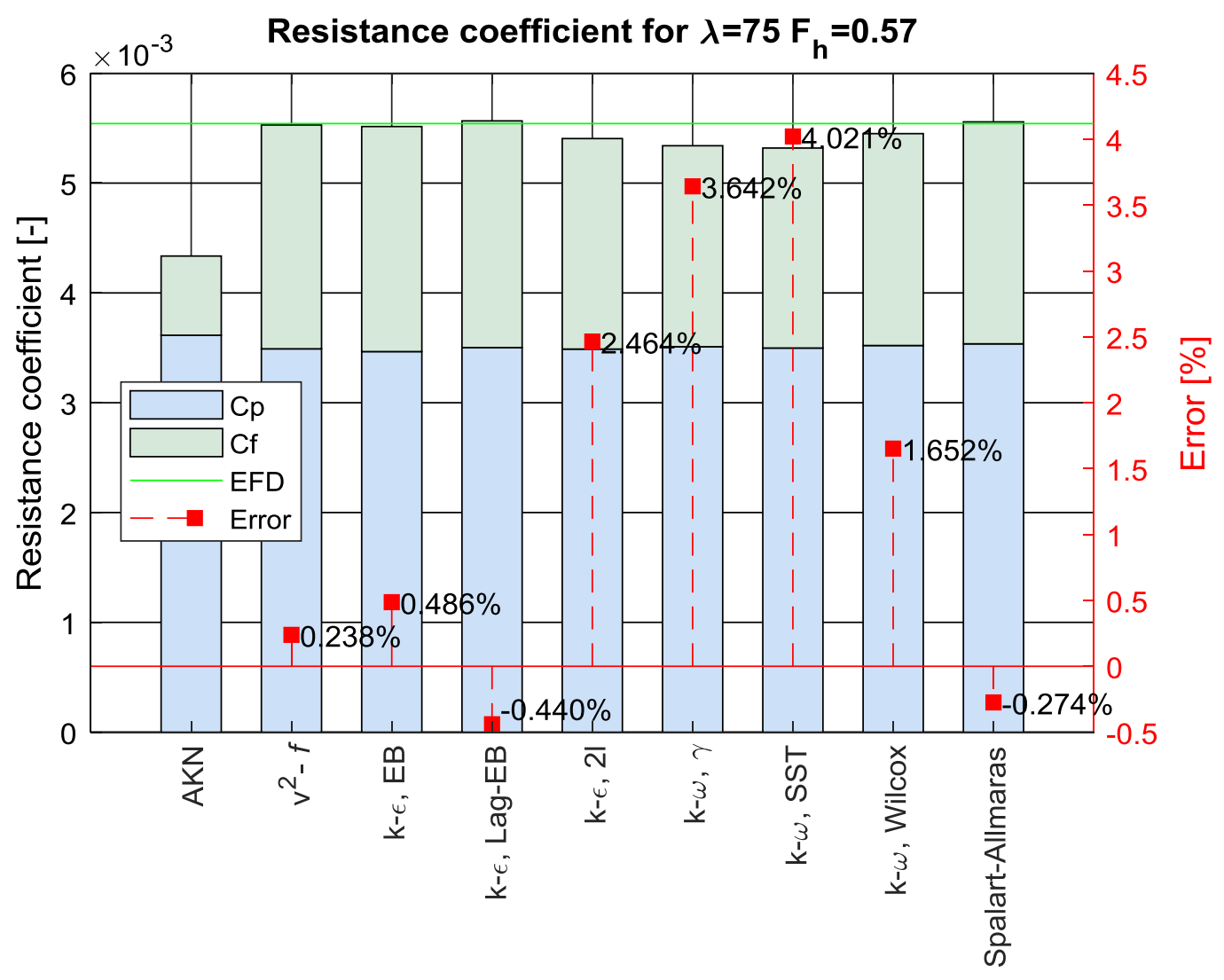


Figure 7. Resistance coefficient comparison for case 2.

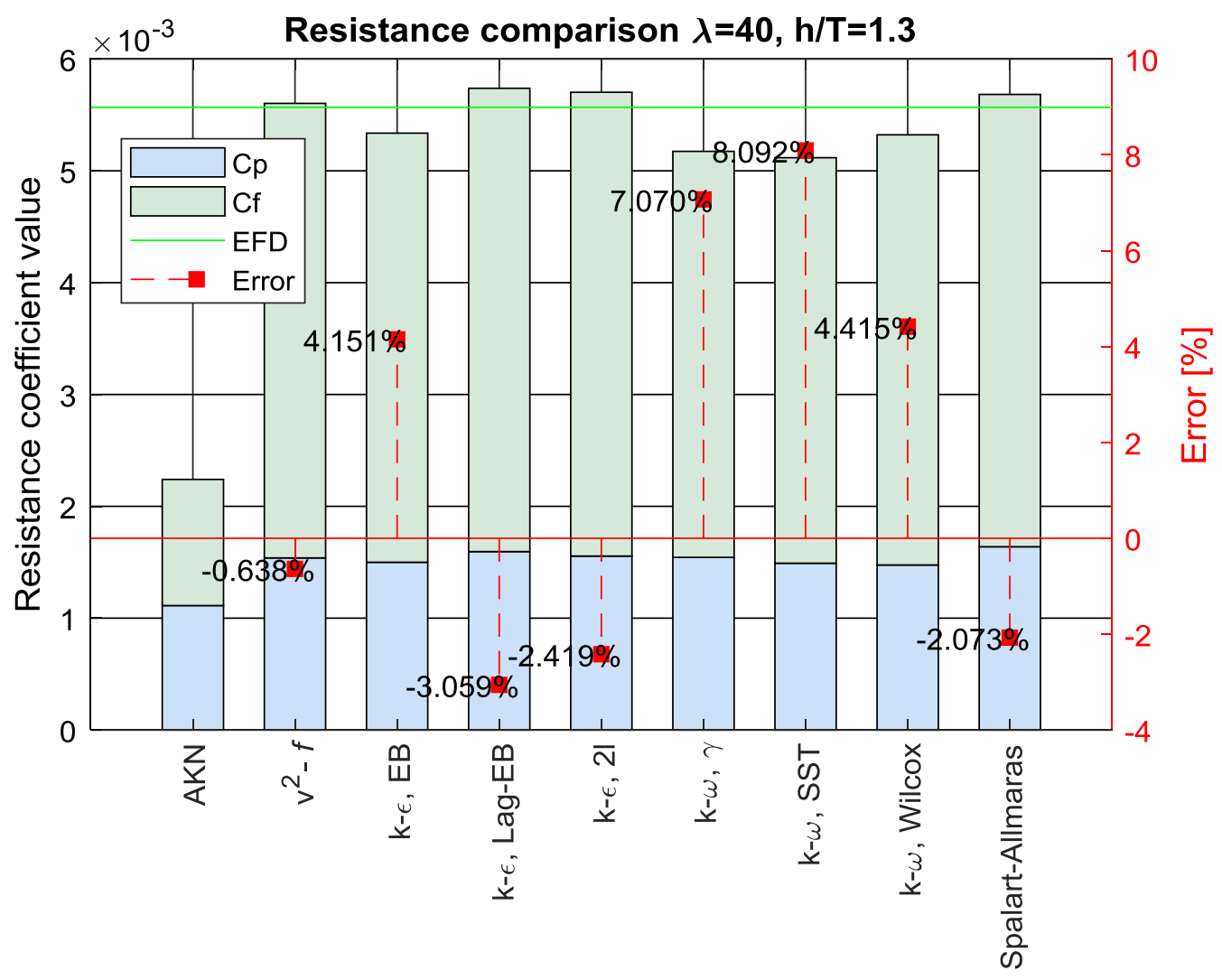

Figure 8. Resistance coefficient comparison for case 3.

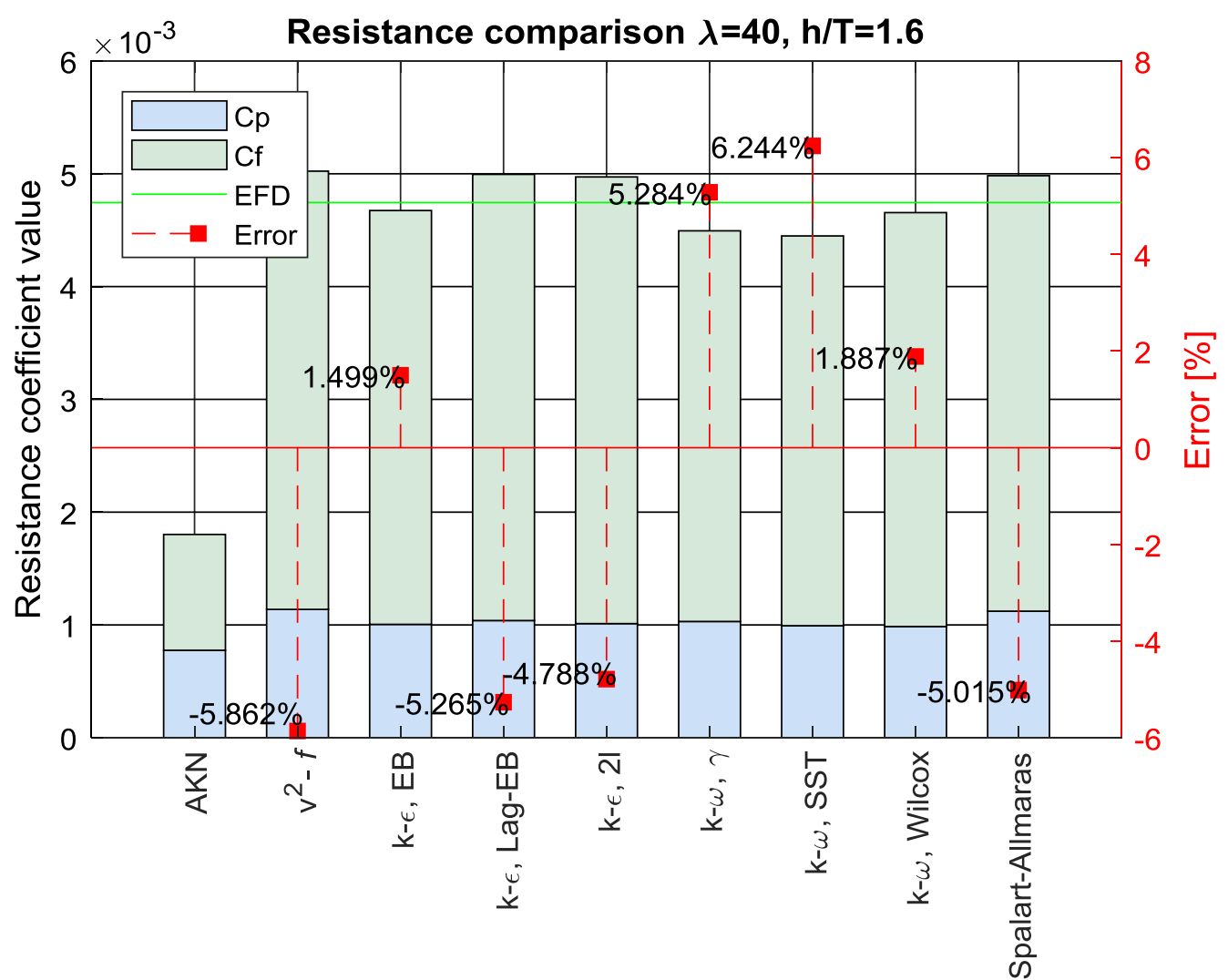


Figure 9. Resistance coefficient comparison for case 4.

Traditionally, ship resistance is extrapolated from model to full-scale following a towing tank experiment (Molland et al., 2017). Once the total resistance has been obtained, it is decomposed into a frictional component, a component due to wave making, and a viscous component. The assumption is that viscous and wave resistance components remain constant with scale, while friction varies as prescribed by the friction line of choice (Grigson, 1999; ITTC, 1999; Katsui et al., 2005). The findings presented herein suggest the wave resistance (if assumed constant with scale) can be established with sufficient accuracy regardless of the turbulence model employed at a high scale factor, where the computational expense is small (refer to Table 4 for cell numbers). Finally, the well-established trend of increase in pressure resistance with decreasing depth is observed in Figure 8 and Figure 9.

One way to confirm that wave resistance is predicted well is to examine whether any differences are present in the location of the waterline along the hull. In this respect, in Figure 10, all wave-hull profiles are shown. The first observation made here is the agreement established between all turbulence models. The $A K N$ closure seems to deviate slightly in cases 1 and 3. In case 2, the $k$ - $\omega S S T$ model diverges from the other turbulence models near the stern of the ship. Furthermore, it exhibits a slight oscillatory behaviour in case 4, not predicted by other closures. Indeed Table 5 can be consulted to confirm the presence of a systematic deviation in the predicted resistance values obtained via the $k$ - $\omega$ SST model. This is surprising considering its popularity among the ship hydrodynamics research community. 

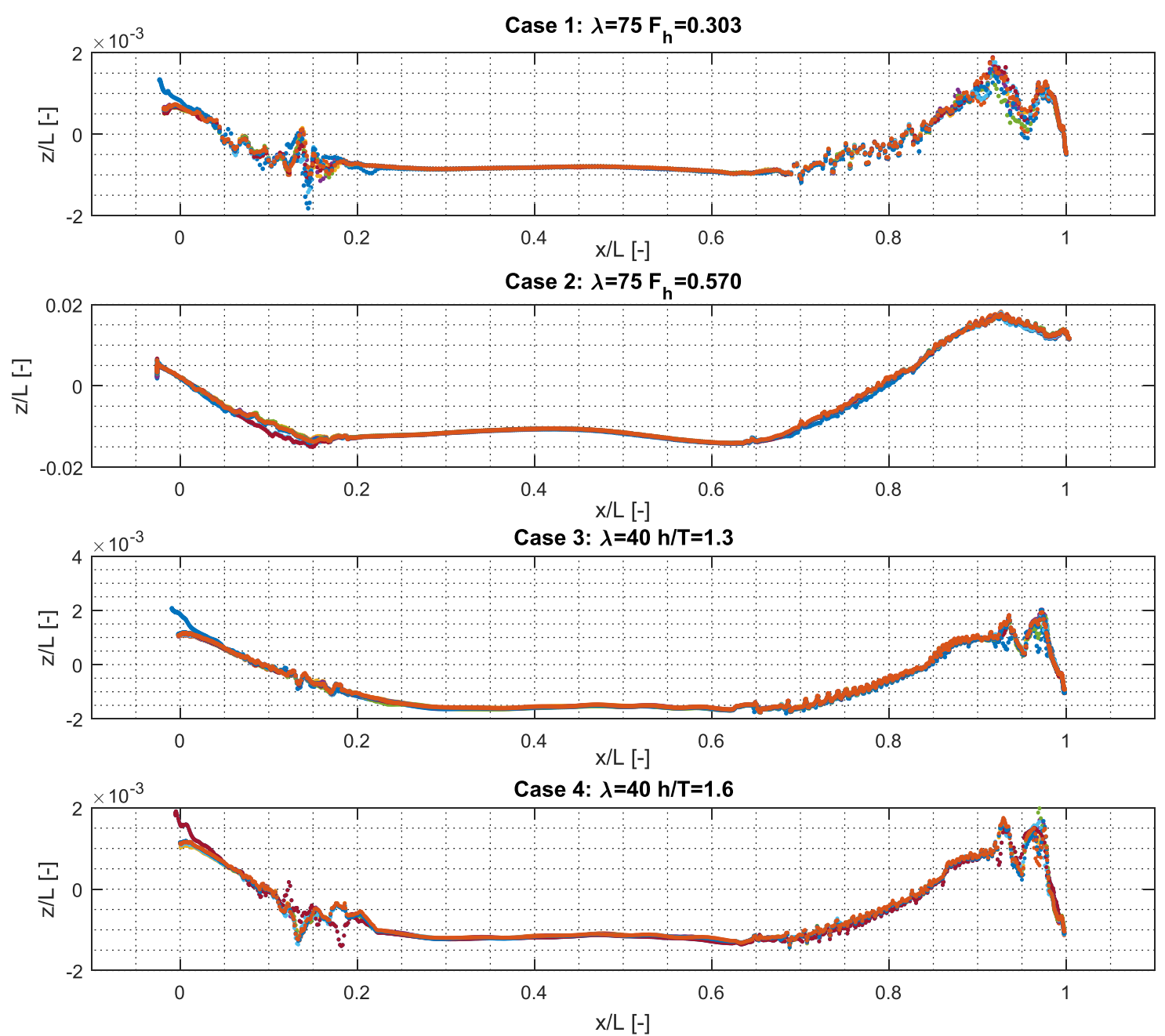

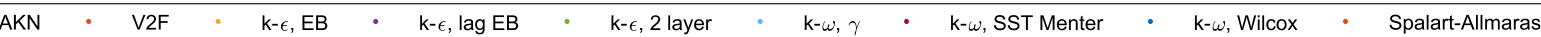

Figure 10. Free surface profile along the hull for all case-studies.

\subsubsection{Friction resistance}

The difference between the smallest and largest skin friction coefficient prediction, excluding $A K N$, is $2.7 \times 10^{-3}$ for cases 1 and 2 , and $2.9 \times 10^{-3}$ for cases 3 and 4 . This an interesting observation, because it serves to highlight that depending on the turbulence model, vastly different results can be obtained in terms of friction. Naturally, this has a strong influence on the total resistance, since friction is one of the main contributing components. Keeping in mind the $y^{+}$distribution shown in Figure 4 (Section 4.3.1), it seems unlikely that predictions made by different turbulence models will collapse into a single value were the grid to be refined further. The skin friction coefficients are graphically compared to established friction lines in Figure 11. With the exception of the two variants based on the SST model ( $k-\omega S S T$, and $k-\omega$ $\gamma)$, the closures predict a significantly higher skin friction than any friction line would suggest. This can be used to highlight the shortcomings of form factor and wave resistance extrapolation techniques, since a reliable frictional resistance is integral to the procedure (Terziev et al., 2019). Confidence in the predictions can be established due to their systematic predictions, as shown in Figure 11, in terms of their relative location on the plot. To elaborate, $k-\omega S S T$, and $k-\omega \gamma$ models are consistently the lowest predictions, whereas $k-\varepsilon$ Lag-EB - the highest. 
There are several likely sources of error in the solutions presented in this work associated with the RANS technique. Stern et al. (2006) identify these errors as turbulence modelling (which is being addressed in the present study), artificial compressibility (also a part of some turbulence models), domain size (dictated by the experimental facilities), and round off errors. It is generally accepted that the latter are negligible. In Section 5.1.2 the numerical uncertainty was bound for an example test case. This allows for the turbulence model to be identified as the dominant contributor to all errors found in the present study.

In terms of $\mathrm{C}_{\mathrm{F}}$, Fukagata et al. (2002) derived a relationship between skin friction and the Reynolds stress distribution across a surface. Their results confirm experimental observations suggesting that the Reynolds stress within 80 wall units $\left(y^{+}<80\right)$ accounts for the vast majority of skin friction (up to 90\%). This region is viscosity-dominated and coincides with the location where turbulence modelling can have the greatest impact. Indeed, one of the aims of turbulence modelling is to predict a Reynolds stress. The three-dimensionality of ship boundary layers is also a critically important fact, since closures are calibrated for two dimensions in almost all cases. In fact, the skin friction is known to consist of pressure development, laminar contribution, spatial development, and Reynold stress components (Stroh, 2016). Shallow waters amplify the three-dimensionality of boundary layer, which was one of the motivating factors cited in the adoption of the case-studies.

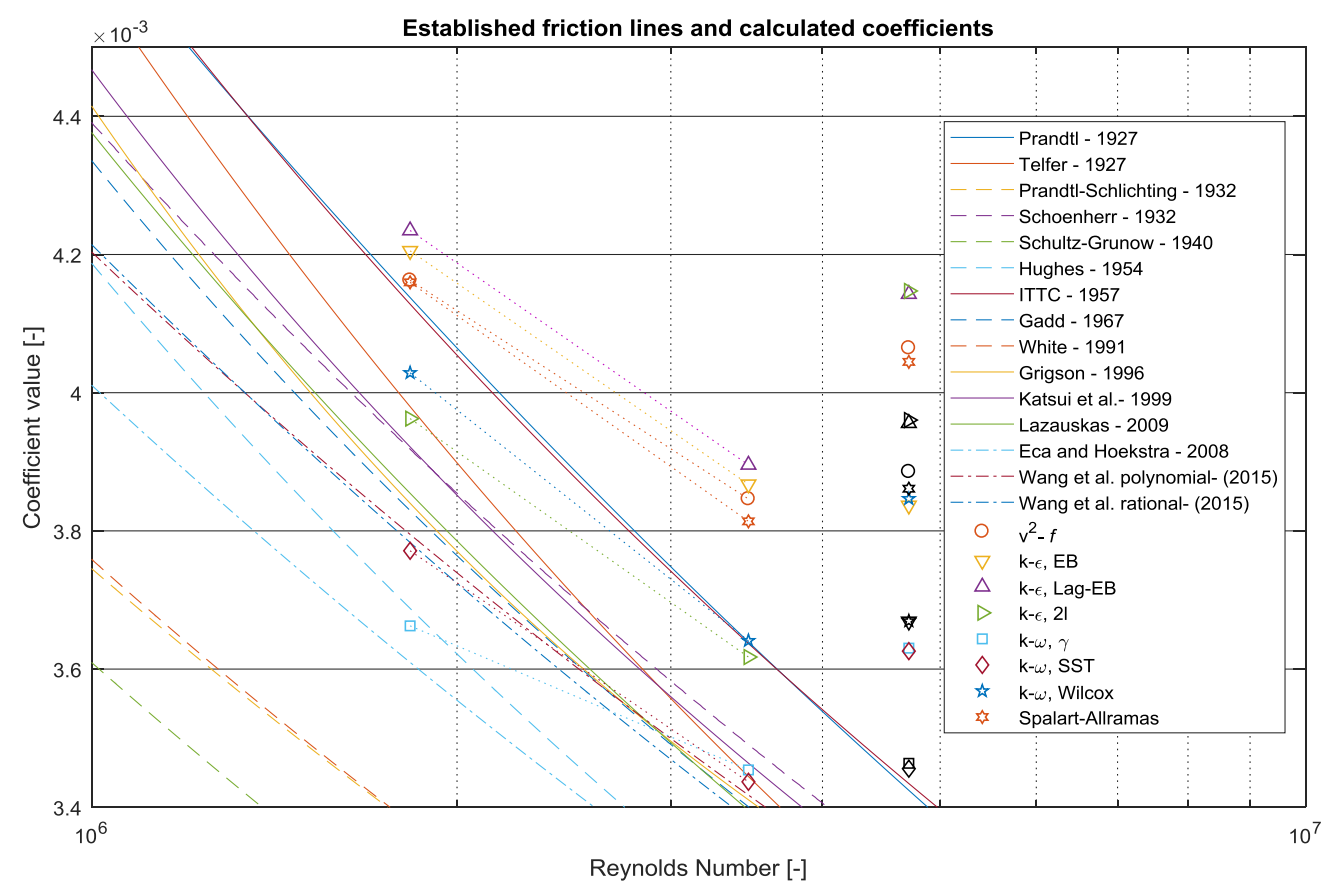

Figure 11. Skin friction comparison. Shapes connected via dotted lines correspond to cases 1 and 2. Solitary coloured shapes correspond to case 3, while the black shapes - case 4 .

Finally, the results shown in Figure 11 suggest that a change in water depth can have influence skin friction substantially. The mechanism by which this occurs is not well understood. Zeng et al. (2018) presented a modification of the ITTC friction line for shallow waters (ITTC, 2017). Their derivation is based on flat plates, and is only applicable to the flat region of a ship's bottom, whereas the friction line proposed by Katsui et al. (2005) is used for the remainder of the wetted area. There are obvious problems with this, mainly due to the fact that the KCS was 
allowed to sink and trim, rendering the boundary layer on the flat bottom highly threedimensional, unlike that of a flat plate. The flow is also forced past the sides of the ship in virtually all shallow water cases, which avoids the violation of the Bernoulli principle. Figure 12 depicts the boundary layer and wake generated by the KCS in case 4 . The top half of the figure was generated to represent $90 \%$ of the free stream velocity $\left(V_{90 \%}=0.657 \mathrm{~m} / \mathrm{s}\right)$., while the bottom half $-99 \%$ of the free-stream velocity $\left(V_{99 \%}=0.7227 \mathrm{~m} / \mathrm{s}\right)$. Figure 12 shows the boundary layer is highly three-dimensional, especially near the bow and stern, where the $99 \%$ free-stream iso-surface is in contact with the seabed over a significant area, where the dynamic pressure is used to colour the surfaces. This is done to highlight the differences in pressure, acting longitudinally and transversely due to the proximity of the seabed. Finally, the waves resulting from the disturbance, caused by the ship also register in the plot.

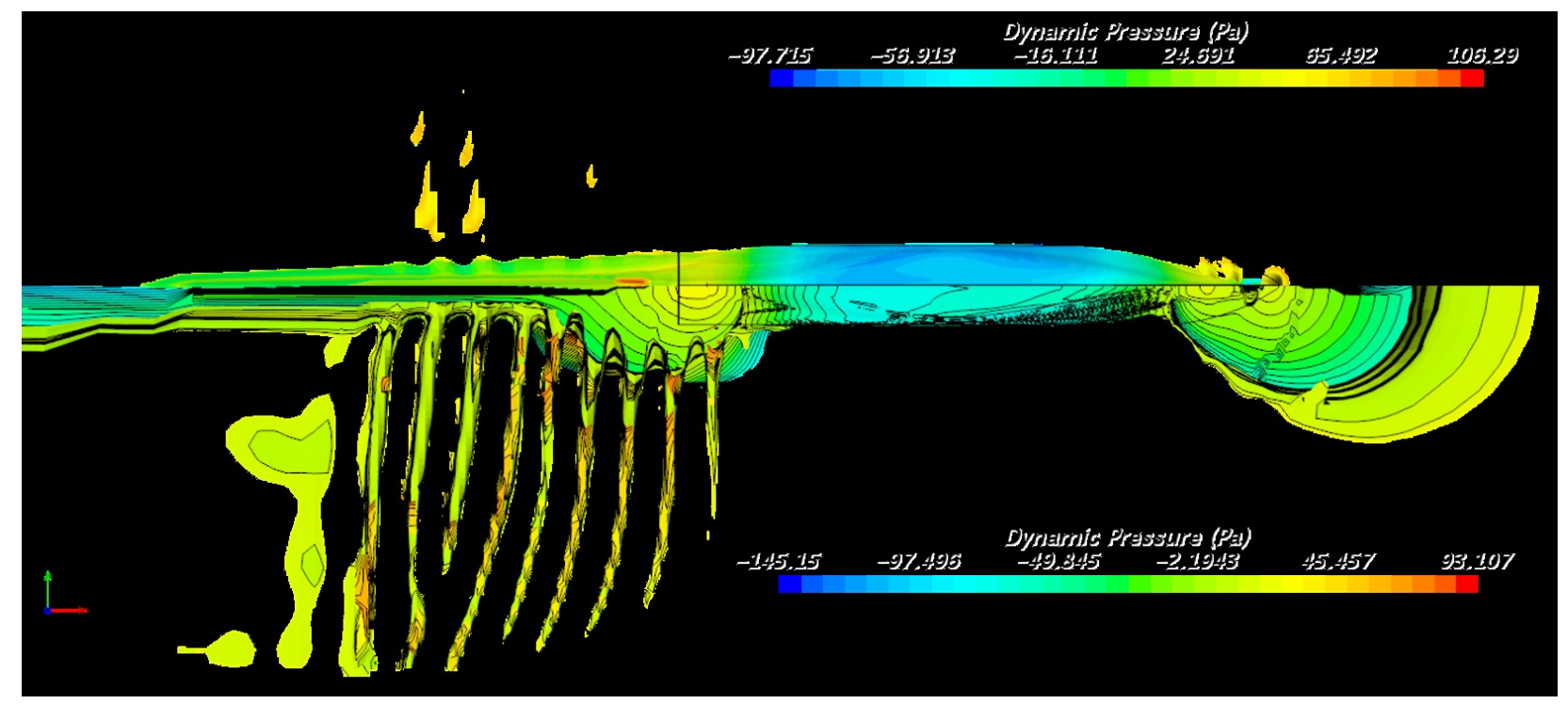

Figure 12. Boundary layer and wake (top view), depicted: case 4. Top: iso-surfaces for $0.9 \times \mathrm{V}$. Bottom: iso-surfaces for $0.99 \times \mathrm{V}$.

\subsubsection{An attempt to identify the optimum turbulence model}

The problem of consistency can be addressed via a modified Youden (1972) plot. Although the original purpose of the technique proposed by the abovementioned author was to establish experimental biases, it can also be used to provide an indication of accuracy and consistency. The required modification is that instead of dimensional quantities, plotted in the $x$ and $y$ axes, we will use the error, calculated in Section 5.1.1, Table 5.

The modified technique begins by plotting the error for each parameter in an $x-y$ plane. As shown in Figure 13, the $y$-axis is chosen to represent error in resistance as calculated for casestudies 2 and 4, whereas the $x$-axis: the error in case-studies 1 and 3 . Thus, the empty shapes in Figure 13 represent the points with coordinates $[x, y]=\left[\mathrm{E}_{1}, \mathrm{E}_{2}\right]$ where the subscripts refer to case-study number, and $\mathrm{E}$ is the error. The filled shapes correspond to points with coordinates $\left[\mathrm{E}_{3}, \mathrm{E}_{4}\right]$. For example, the empty blue circle $\left(v^{2}-f\right.$ model $)$ in Figure 13 has an $x$ - coordinate equal to the total resistance error for case 1 (3.74\%), and a - coordinate equal to the total resistance error for case $2(0.24 \%)$, and a $y$-coordinate corresponding to the error in case 2 . Similarly, the filled blue circle has $x$ - coordinates equal to the $\mathrm{C}_{\mathrm{T}}$ error for case 3 , and $y-$ coordinate equal to $\mathrm{C}_{\mathrm{T}}$ error for case 4 . 
As described by Youden (1972), the next step is to construct the straight lines, representing the median $x$ and $y$ values. Depending on the location of each point within the plot, a systematic bias, or lack thereof can be established. For instance, a point lying in the first quadrant (the standard convention is adopted), established via the intersection of the median values, suggests a systematic overprediction. Using the intersection of the median $x$ and $y$ values, a 45 degree diagonal is drawn. This diagonal can be useful in giving a measure of consistency each turbulence model provides in its predictions. In other words, points close to the diagonal perform in a similar manner in all four case-studies, i.e. systematically. An extra layer of information can be extracted due to the fact that we have made use of the error in Figure 13.

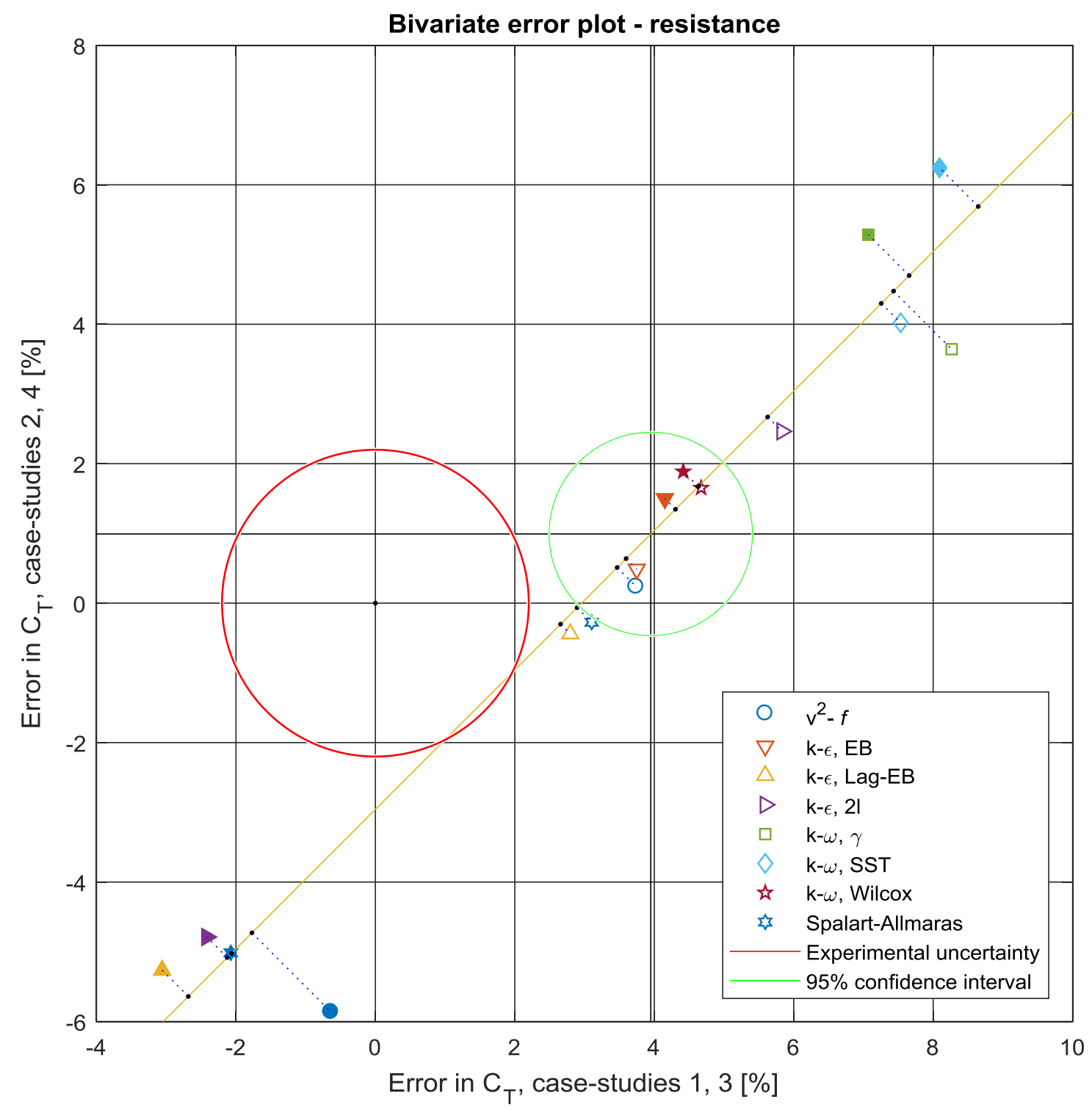

Figure 13. Bivariate error plot for resistance.

By requiring that the error is the metric against which the points are assessed, we can simultaneously take into account their performance against the relevant experiment. An additional consequence of our choice is that we can directly draw the experimental uncertainty onto the plot. For case-studies 1 and 2, this is $2.20 \%$. For cases 3 and 4, the uncertainty is not reported, therefore, we will assume it has the same magnitude as in cases 1 and 4 . Armed with this information, a circle, centred at $[0,0]$, with a radius of 2.20 can be drawn, giving a graphical 
interpretation of the error and biases in all four cases (the red circle in Figure 13). If all experimental uncertainties were known, an ellipse would be required, as is the case with modified versions of the plot (Velázquez and Asuero, 2017).

The mathematics behind different turbulence models cannot provide us with any information we could use to forecast whether the values will under or overpredict the experiment prior to running the simulation. If all turbulence models were made equal, we would expect the values to be scattered evenly in each quadrant around the median $x-y$ intersection. This is true because all models are calibrated against a similar set of experiments, and therefore should be equally robust. Therefore, displacement along the diagonal can be interpreted as bias, whereas offset from the diagonal is an indication of erratic behaviour.

The green circle, centred at the intersection of the median $x$ and $y$ values in Figure 13, represents the $95 \%$ confidence level, derived following the procedure of Youden (1972). A high, simultaneous deviation from both circles (representing the experimental uncertainty and confidence level) indicates a turbulence model's inability to cope well with the case it is applied to. Thus, the proximity of $k-\omega$ Wilcox, and $k-\varepsilon E B$ (and its Lag extension) models, coupled with their small distance from the diagonal, indicates they perform well, consistently, and reliably. By contrast, the $k-\varepsilon 2 l$ model shows erratic behaviour, over and underpredicting results by a large margin depending on the case-study. Thus, the results suggest that applying this closure, the user cannot hope to predict with any confidence what the outcome of a simulation will be. If either of the well-performing models mentioned above were to be used, one could expect to achieve results with a small, positive error. That is, provided the numerical set-up is reasonably similar to that presented herein.

Unfortunately, Figure 13 takes into account only resistance. Thus, no information regarding the turbulence models' behaviour in sinkage and trim can be deduced from it. For this reason, Figure 14 was constructed using case-studies 1, 2 and 4. On the $x$-axis, the error in sinkage is plotted, while on the $y$-axis - the corresponding case-study's error in resistance. As before, the empty shapes represent case 1 , the filled shapes - case 2 , and the black shapes - case 4 . Case 3 is omitted due to the large errors in sinkage, which would push the $x$ scale too far for any meaningful discussion. 


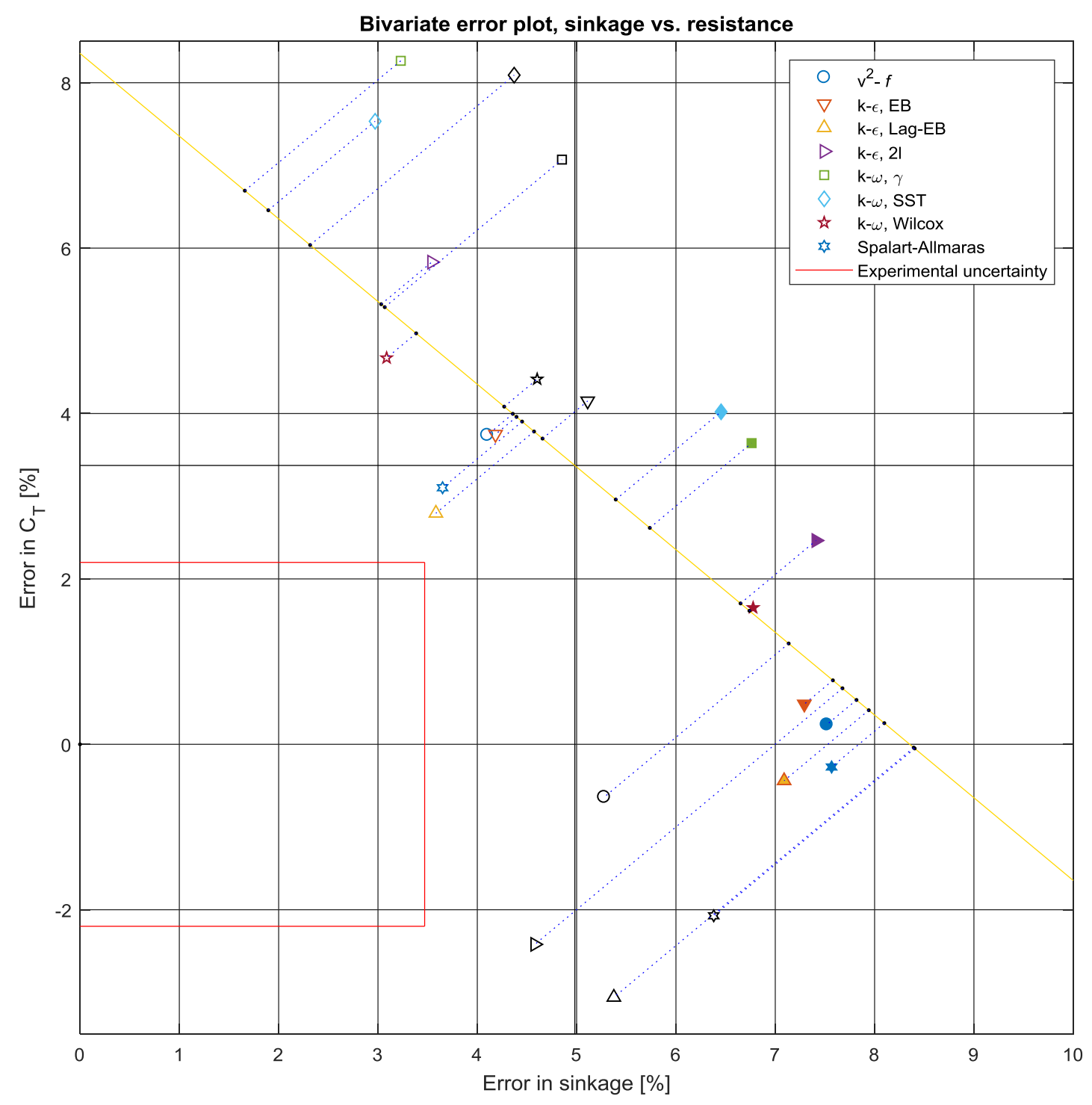

Figure 14. Bivariate error plot: sinkage vs. resistance.

A consequence of the arrangement of the points is that the diagonal, constructed from the intersection of the median $x$ and $y$ values has a negative slope. Since different experimental uncertainties are reported in Elsherbiny et al. (2019) for each axis, the shape constructed in Figure 14 is a rectangle with dimensions $3.47 \times 2.2$, matching the uncertainties in sinkage and resistance, respectively. As before, the lack of reported data for case-studies 3 and 4 means a uniform cross-experimental uncertainty is assumed.

All turbulence models seem to follow certain tendencies in Figure 14, where $k-\varepsilon$ type models tend to fall beneath the diagonal, while $k-\omega$ based models are located above it. A notable exception to this is the $k-\varepsilon 2 l$ model, which has two points above, and one below the diagonal. Figure 14 happens to be constructed so that points below the diagonal are closer to the limits of the rectangle, and therefore closer to the experimental uncertainty bounds. Due to the large scatter in values, the $95 \%$ confidence interval, based solely on the points' location has not been constructed.

It is important to keep in mind that the 'distance to the diagonal' metric is purely artificial. However, since it depends on the overall scatter of the predictions, it serves as a good indication 
of erratic behaviour. As alluded to previously, if all turbulence models were equally robust, they would all lie within a small circle, centred at the median $x$ and $y$ values. Large deviations, prevalent to the $k$ - $\varepsilon$-class models, points towards unreliable predictions. This is not in reference to the accuracy itself. Indeed, in some cases their predictions fall within the experimental uncertainty (e.g. $v^{2}-f, E B$, and $\operatorname{Lag} E B$ models in resistance for case 2). Instead, reliable turbulence models are those that preform similarly across all case-studies they are applied to.

For Figure 14, the set of criteria used to establish a hierarchy of performance follow from the assessment performed for Figure 13. Namely, the quadrant in which each point lies, as well as the distance to the diagonal, and origin are taken into account. These metrics, in addition to their overall Root Mean Square (RMS) values have been constructed graphically in Figure 15, where only the results depicted in Figure 14 are used. Here, the $k$ - $\omega$ Wilcox model exhibits the desired characteristics to greater extent than the remaining candidates. Firstly, the distance to the diagonal is small throughout, relative to other cases. This is an indication the turbulence model is not an outlier in the scatter of predictions. Secondly, the distance to the origin is reasonable. Finally, two out of three points predicted via this model fall within the same $\left(2^{\text {nd }}\right)$ quadrant, indicating a degree of consistency.

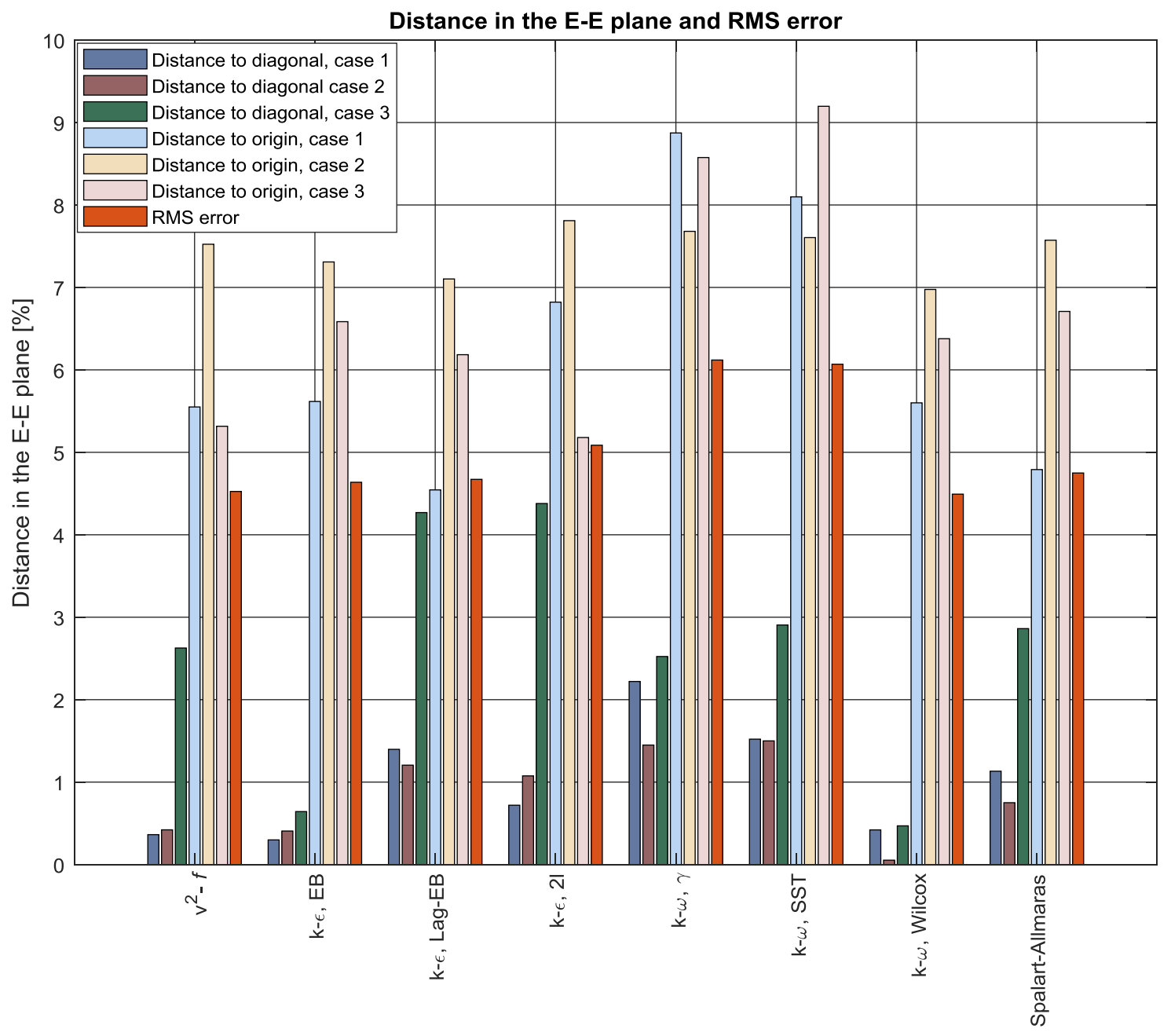

Figure 15. Distances in the Error-Error plane and RMS error for case-studies 1-3.

The $k-\varepsilon E B$ model performs marginally worse, with all three points within different quadrants. Other strong candidates include the $v^{2}-f$ model, whose predictions tend to lie close to those 
obtained via the $k-\varepsilon E B$ closure. Perhaps surprisingly, the results suggest that we should rule out using the two models accounting for over $80 \%$ of all research done in the field of ship hydrodynamics (based on the dataset established earlier). Namely, the $k-\varepsilon 2 l$ and $k-\omega S S T$ models (see Figure 1 in Section 2). On the other hand, the Spalart Allmaras model performs almost as well as the more complex, recent closures, such as $k-\varepsilon E B$ and Lag-EB models. In part, we have echoed the findings of Eça et al. (2018), who found that for the $k$ - $\omega S S T$ model, a $y^{+} \approx 0.1$ is required to achieve the same level of accuracy as other turbulence models. This requirement restricts the use of the $k-\omega S S T$ closure, because of the difficulties related to the constraint. Specifically, the cell numbers would increase substantially, as well as the their aspect ratio, which compromises the solution's stability and convergence properties (Eça et al., 2015; ITTC, 2011).

\subsubsection{Quantification of the computational resource}

The final metric, against which turbulence models are evaluated is time per iteration. Although the solver's time per iteration has been recorded throughout the entire duration of the simulation, only values achieved during the first 5 seconds are reported. This is done because once the ship is allowed to translate and rotate, the time history is contaminated with the numerical algorithms responsible for doing this. Additionally, the auto save function of Star$\mathrm{CCM}+$ was employed to routinely save the simulation (every 5 seconds of physical time), which registers as a spike in the time per iteration monitor. Therefore, global averages would not be a suitable metric.

All simulations were performed using the high performance computer facilities at the University of Strathclyde, Archie-WeSt. The facility features 2 Intel Xeon Gold 613820 core 2.0GHz CPUs, with 40 cores per node, and 192 GB RAM allocated per node. To run the simulations described herein, one node (with 40 cores) was used per case per turbulence model (i.e. per simulation). The resulting time per iteration is shown graphically in Figure 16 for case 1. It should be stressed that all results presented in Figure 16 are highly dependent on the solver employed. The relationship between different closures would most likely be maintained if carried forward to a case using Star-CCM+ featuring different cell numbers. However, scalability is not uniform across all RANS solvers, as discussed by Robertson et al. (2015).

The relationship between core numbers and efficiency in obtaining the solution is known to be inverse in nature (Axtmann and Rist, 2016). Therefore, as the cell numbers increase, the difference in computational time will likely decrease. For this reason, case 1, with the smallest number of cells, is chosen for this assessment. In this respect, the development of algorithms tasked with optimising parallelisation is an active field of research, although its relationship with DNS is stronger than RANS approaches (Cifani et al., 2018; Kooij et al., 2018; van der Poel et al., 2015). 


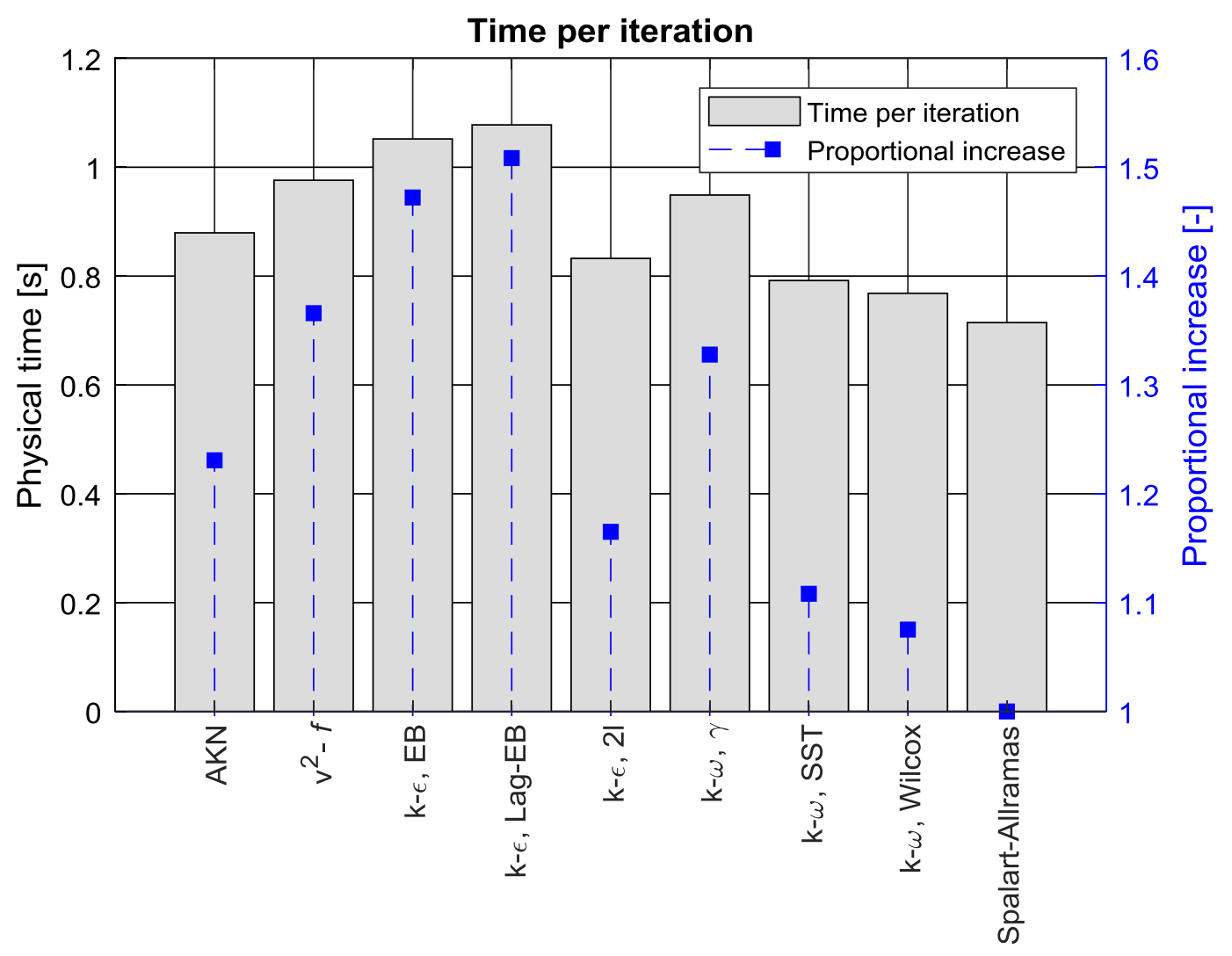

Figure 16. Time per iteration for case 1 .

As expected, the Spalart-Allmaras model provides the smallest computational time requirements, and is used to establish a proportional increase for all other models. The $k-\omega$ Wilcox model provides the smallest time after the Spalart-Allmaras closure, closely followed by $k-\omega S S T$. All $k-\varepsilon$ variants exhibit a higher time per iteration due to the additional near-wall treatments or extra equations introduced. For instance, the main source of differences between $k-\omega$ Wilcox and $k-\varepsilon 2 l$ in terms of time is the realizability requirements for the latter. On the other hand, the imposition of a $\gamma$ transition is shown to increase substantially the computational requirements, while providing little, if any improvements in terms of accuracy, consistency or reliability of the solution. Furthermore, introducing additional equations into the turbulence closure implicitly destabilises the solution. Specifically, more ways in which the numerical calculation could fail are introduced. This is sometimes thought of as numerical stiffness of the solution, and was the reason given for employing a $2^{\text {nd }}$ order convection scheme, rather than the more accurate $3^{\text {rd }}$ order option. In this respect, the practitioner must have a sense of the accuracy, consistency, and computational resource required. Improving the latter is normally associated with a detrimental effect in the former two. In this study, the results suggest this trade-off may not always be necessary. That is, provided a suitable numerical set-up is employed.

One final aspect requiring discussion is associated with the solver itself. Research has shown that it is dangerous to generalise outcomes achieved in one software to another. Specifically, the same mesh and set-up can lead to different solutions depending on the solver employed. This problem was highlighted by Iaccarino (2002), who found significant deviations across RANS software. In consequence, the hierarchy established in this paper would, in all likelihood, differ if repeated using a different solver. This is true because software providers 
do not disclose all information related to the algorithms and sub-routines used. It is therefore not possible to predict what the effect of replicating this study elsewhere would have.

\section{Concluding remarks and future work}

This study has focused on reducing the uncertainty in turbulence model selection for a class of problems in ship hydrodynamics. Nine turbulence models were identified as potential candidates for the assessment based on a literature survey. Emphasis was placed on devising a hierarchy depending on performance, since it is not possible to use any other meaningful metric. The adopted case-studies, attempting to replicate a set of shallow water experiments, were specifically selected to challenge the turbulence models.

We demonstrated the validity of the implemented numerical set-up by validating the solutions against experimental data and by estimating the numerical uncertainty for an example casestudy. As a piece of future work, all turbulence models should be subjected to the numerical verification procedure described in Section 5.1.2. Citing the inordinate computational expense as primary justification, the procedure was not thoroughly completed herein. However, doing so would improve on the current understanding of modelling errors induced by the type $f$ closure. As such, this has been identified as a piece of future research.

The results indicate that pressure resistance and its constituent components are predicted well by all turbulence models. This finding has wider implications in the extrapolation of ship resistance from model to full-scale, as it implies wave resistance is largely independent of turbulence modelling. Friction resistance was shown as the main source of errors. There are several identified areas contributing to the observed discrepancies, other than those due to the numerical set-up, which are bound within acceptable levels. While turbulence models tend to be calibrated against two dimensional cases, a strong three-dimensional boundary layer is observed in the investigated cases.

The observed difference between the highest and smallest frictional resistance prediction was calculated to be nearly constant across all case-studies. This indicates that a strong turbulence modelling sensitivity should be prevalent for full-scale computations, as forecasted by Duvigneau et al. (2003). Our difficulties in creating sufficiently fine meshes to resolve the boundary layer at full-scale is also a suspected contributor to the here-described uncertainty. Specifically, the present study can be said to have established a hierarchy for similar numerical set-ups. If the $y^{+}$values are varied significantly, the ranking establishing in this study could well be different.

In an attempt to rank turbulence models with respect to their accuracy and consistency, the $k$ $\omega$ Wilcox and elliptic blending models showed best performance. The assessment was carried out via a modified Youden bivariate plot, where the error in relation to the experimental values was plotted on both axes. The performance criteria included are thought to be capable of providing an initial estimate of the outcome prior to completing the simulation. Coupling these findings with the time per iteration characteristics of each turbulence model suggests the optimal choice is the $k-\omega$ Wilcox turbulence model for bare hull ship resistance computations.

In this study, the action of a rotating propeller and surface roughness have been neglected. These can undoubtedly alter the results observed. Their omission is thought to be justified because, in the latter case, a factor can be applied to represent the roughness. In the case of propellers, the definition of a separate region in a RANS solver to perform the required 
rotations allows the user to modify the physics within it. Therefore, provided the optimum turbulence model for propellers is known, it seems straightforward to prescribe the relevant characteristics where necessary. These must therefore be investigated in a similar manner to what has been done for bare hull resistance, sinkage, and trim in this study, which is left as a piece of future work.

\section{Acknowledgements}

Results were obtained using the ARCHIE-WeSt High Performance Computer (www.archiewest.ac.uk) based at the University of Strathclyde. The work reported in this paper is drawn from the first author's PhD thesis. The first author gratefully acknowledges the scholarship provided by the Faculty of Engineering at the University of Strathclyde, which fully supports his $\mathrm{PhD}$.

\section{Data statement}

All data underpinning this publication are openly available from the University of Strathclyde KnowledgeBase at https://doi.org/10.15129/0650963a-1aea-4ecc-9611-79f7c551ddf2 


\section{References}

Abe, K., Kondoh, T., Nagano, Y., 1994. A new turbulence model for predicting fluid flow and heat transfer in separating and reattaching flows-1. Flow field calculations. Int. J. Heat Mass Transf. 37, 139-151. https://doi.org/10.7566/JPSJ.84.054710

Abu-Ghannam, B.J., Shaw, R., 1980. Natural Transition of Boundary Layers - The Effects of Turbulence, Pressure Gradient, and Flow History. J. Mech. Eng. Sci. 22, 213-228. https://doi.org/https://doi.org/10.1243/JMES_JOUR_1980_022_043_02

Argyropoulos, C.D., Markatos, N.C., 2015. Recent advances on the numerical modelling of turbulent flows. Appl. Math. Model. 39, 693-732. https://doi.org/10.1016/j.apm.2014.07.001

Axtmann, G., Rist, U., 2016. Scalability of OpenFOAM with Large EddySimulations and DNS on High-PerformanceSystems, in: Nagel W., Kröner D., Resch M. (Eds) High Performance Computing in Science and Engineering '16. Springer, Cham. https://doi.org/10.1109/CAHPC.2005.27

Bailly, C., Comte-Bellot, G., 2004. Turbulence, Springer. Springer, Cham. https://doi.org/https://doi.org/10.1007/978-3-319-16160-0

Baldwin, B., Timothy, B., 1990. A one-equation turbulence transport model for high Reynolds number wall-bounded flows, National Aeronautics and Space Administration. https://doi.org/10.2514/6.1991-610

Baldwin, B.S., Lomax, H., 1978. Thin Layer Approximation and Algebraic Model for Separated Turbulent Flows, In 16th aerospace sciences meeting.

Beck, R.F., 1977. Forces and Moments on a Ship Moving in a Shallow Channel. J. Sh. Res. 21, 107-119.

Beck, R.F., Newman, J.N., Tuck, E.O., 1975. Hydrodynamic forces on ships in dredged channels. J. Sh. Res. 19, 166-171.

Bhushan, S., Alam, M.F., Walters, D.K., 2013. Evaluation of hybrid RANS/LES models for prediction of flow around surface combatant and Suboff geometries. Comput. Fluids 88, 834-849. https://doi.org/10.1016/j.compfluid.2013.07.020

Billard, F., Laurence, D., 2012. A robust k- $\varepsilon-v 2 / k$ elliptic blending turbulence model applied to near-wall, separated and buoyant flows. Int. J. Heat Fluid Flow 33, 45-58. https://doi.org/10.1016/j.ijheatfluidflow.2011.11.003

Blasius, H., 1908. The boundary layers in fluids with little friction.

Cakici, F., Sukas, O.F., Kinaci, O.K., Alkan, A.D., 2017. Prediction of the vertical motions of the dtmb 5415 ship using different numerical approaches. Brodogradnja 68, 29-44. https://doi.org/10.21278/brod68203

Carrica, P.M., Huang, J., Noack, R., Kaushik, D., Smith, B., Stern, F., 2010. Large-scale DES computations of the forward speed diffraction and pitch and heave problems for a surface combatant. Comput. Fluids 39, 1095-1111. https://doi.org/10.1016/j.compfluid.2010.02.002

Carrica, P.M., Wilson, R. V., Noack, R.W., Stern, F., 2007. Ship motions using single-phase level set with dynamic overset grids. Comput. Fluids 36, 1415-1433. 
https://doi.org/10.1016/j.compfluid.2007.01.007

Cazalbou, J.B., Spalart, P.R., Bradshaw, P., 1994. On the behavior of two-equation models at the edge of a turbulent region. Phys. Fluids 6, 1797-1804.

https://doi.org/10.1063/1.868241

Celik, I.B., Ghia, U., Roache, P.J., Freitas, C.., 2008. Procedure for Estimation and Reporting of Uncertainty Due to Discretization in CFD Applications. J. Fluids Eng. 130, 078001. https://doi.org/10.1115/1.2960953

Chen, H.C., Patel, V.C., 1988. Near-wall turbulence models for complex flows including separation. AIAA J. 26, 641-648. https://doi.org/10.2514/3.9948

Chien, K.Y., 1982. Predictions of Channel and Boundary-Layer Flows with a Low-ReynoldsNumber Turbulence Model. AIAA J. 20, 33-38. https://doi.org/10.2514/3.51043

Chou, P.Y., 1945. On velocity correlations and the solutions of the equations of turbulent fluctuation. Q. Appl. Math. 3, 38-54. https://doi.org/10.1090/qam/11999

Cifani, P., Kuerten, J.G.M., Geurts, B.J., 2018. Highly scalable DNS solver for turbulent bubble-laden channel flow. Comput. Fluids 172, 67-83. https://doi.org/10.1016/j.compfluid.2018.06.008

Darrigol, O., 2017. Joseph Boussinesq's legacy in fluid mechanics. Comptes Rendus - Mec. 345, 427-445. https://doi.org/10.1016/j.crme.2017.05.008

Darrigol, O., Turner, J.S., 2006. Worlds of flow: A history of hydrodynamics from the bernoullis to prandtl, Physics Today. https://doi.org/10.1063/1.2349735

Davidson, L., Nielsen, P. V, Sveningsson, A., 2003. Modifications of the V2F Model for Computing the Flow in a 3D Wall Jet. Turbul. Heat Mass Transf. 4, 577-584.

Deng, R., Li, C., Huang, D., Zhou, G., 2015. The Effect of Trimming and Sinkage on the Trimaran Resistance Calculation, Procedia Engineering. Elsevier B.V. https://doi.org/10.1016/j.proeng.2015.11.199

Durbin, P.A., 2017a. Some Recent Developments in Turbulence Closure Modeling. Annu. Rev. Fluid Mech. 50, 77-103. https://doi.org/10.1146/annurev-fluid-122316-045020

Durbin, P.A., 2017b. Perspectives on the Phenomenology and Modeling of Boundary Layer Transition. Flow, Turbul. Combust. 99, 1-23. https://doi.org/10.1007/s10494-017-98199

Durbin, P.A., 1996. On the k-3 stagnation point anomaly. Int. J. Heat Fluid Flow 17, 89-90. https://doi.org/10.1016/0142-727X(95)00073-Y

Durbin, P.A., 1995. Separated flow computations with the k-epsilon-v-squared model. AIAA J. 33, 659-664. https://doi.org/10.2514/3.12628

Durbin, P.A., 1993a. Application of a near-wall turbulence model to boundary layers and heat transfer. Int. J. Heat Fluid Flow 14, 316-323. https://doi.org/10.1016/0142727X(93)90004-7

Durbin, P.A., 1993b. A Reynolds stress model for near-wall turbulence. J. Fluid Mech. 249, 465-498. https://doi.org/10.1017/S0022112093001259

Durbin, P.A., 1991. Near-wall turbulence closure modeling without "damping functions." 
Theor. Comput. Fluid Dyn. 3, 1-13. https://doi.org/10.1007/BF00271513

Durbin, P.A., Pettersson Reif, B.A., 2011. Statistical theory and modelling for turbulent flow, Second Edi. ed. Wiley.

Duvigneau, R., Visonneau, M., Deng, G.B., 2003. On the role played by turbulence closures in hull shape optimization at model and full scale. J. Mar. Sci. Technol. 8, 11-25. https://doi.org/10.1007/s10773-003-0153-8

Eça, L., Pereira, F.S., Vaz, G., 2018. Viscous flow simulations at high Reynolds numbers without wall functions: Is $y+\simeq 1$ enough for the near-wall cells? Comput. Fluids 170 , 157-175. https://doi.org/10.1016/j.compfluid.2018.04.035

Eça, L., Saraiva, G., Vaz, G., Abreu, H., 2015. The Pros and Cons of Wall Functions. OMAE2015, St.Johns, Newfoundland, Canada 0-11. https://doi.org/10.1115/OMAE2015-41518

El-Behery, S.M., Hamed, M.H., 2011. A comparative study of turbulence models performance for separating flow in a planar asymmetric diffuser. Comput. Fluids 44, 248-257. https://doi.org/10.1016/j.compfluid.2011.01.009

Elsherbiny, K., Tezdogan, T., Kotb, M., Incecik, A., Day, S., 2019. Experimental analysis of the squat of ships advancing through the New Suez Canal. Ocean Eng. 178, 331-344. https://doi.org/10.1016/j.oceaneng.2019.02.078

EMSA (European Maritime Safety Agency), 2018. Annual Overview of Marine Casualties and Incidents 2018.

EMSA (European Maritime Safety Agency), 2017. Annual overview of marine casualties and incidents 2017.

EMSA (European Maritime Safety Agency), 2016. Annual overview of marine casualties and incidents 2016.

EMSA (European Maritime Safety Agency), 2015. Annual overview of marine casualties and incidents 2011-2015.

Farkas, A., Degiuli, N., Martić, I., 2018. Assessment of hydrodynamic characteristics of a full-scale ship at different draughts. Ocean Eng. 156, 135-152. https://doi.org/10.1016/j.oceaneng.2018.03.002

Fukagata, K., Iwamoto, K., Kasagi, N., 2002. Contribution of Reynolds stress distribution to the skin friction in wall-bounded flows. Phys. Fluids 14, L73-L76. https://doi.org/10.1063/1.1516779

Gorji, S., Seddighi, M., Ariyaratne, C., Vardy, A.E., O’Donoghue, T., Pokrajac, D., He, S., 2014. A comparative study of turbulence models in a transient channel flow. Comput. Fluids 89, 111-123. https://doi.org/10.1016/j.compfluid.2013.10.037

Grigson, C.W.B., 1999. A planar friction algorithm and its use in analysing hull resistance. Trans. RINA.

Haase, M., Zurcher, K., Davidson, G., Binns, J.R., Thomas, G., Bose, N., 2016. Novel CFDbased full-scale resistance prediction for large medium-speed catamarans. Ocean Eng. 111, 198-208. https://doi.org/10.1016/j.oceaneng.2015.10.018

Hirt, C.. W., Nichols, B.. D., 1981. Volume of fluid (VOF) method for the dynamics of free 
boundaries. J. Comput. Phys. 39, 201-225. https://doi.org/10.1016/00219991(81)90145-5

Iaccarino, G., 2002. Predictions of a Turbulent Separated Flow Using Commercial CFD Codes. J. Fluids Eng. 123, 819. https://doi.org/10.1115/1.1400749

ITTC, 2017. Recommended Procedures 1978 ITTC Performance Prediction Method, 4th revision, 7.5 - 0203 - 01.4. 28th Int Towing Tank Conf.

ITTC, 2014. ITTC - Recommended Procedures and Guidelines - Practical guidelines for ship CFD applications. 7.5-03-02-03 (Revision 01). ITTC - Recomm. Proced. Guidel. 19.

ITTC, 2011. Recommended Procedures and Guidelines: Practical Guidelines for Ship CFD. 26th Int. Towing Tank Conf.

ITTC, 2008. Uncertainty Analysis in CFD Verification and Validation Methodology and Procedures. 25th ITTC 2008, Resist. Comm. 12.

ITTC, 1999. 1978 ITTC Performance Prediction Method 7.5 - 02 - 03 - 01.4.

Johnson, D.A., King, L.S., 1984. A mathematically simple turbulence closure model for attached and separated turbulent boundary layers. AIAA J. 23, 1684-1692. https://doi.org/10.2514/3.9152

Jones, D.A., Clarke, D.B., 2010. Fluent Code Simulation of Flow around a Naval Hull: the DTMB 5415. Def. Sci. Technol. Organ. Victoria (Australia), Marit. platforms Div.

Jones, W.P., Launder, B.E., 1972. The Prediction of Laminarization with a Two-Equation Model of Turbulence. Int. J. Heat Mass Transf. 15, 301-314. https://doi.org/10.1016/0017-9310(72)90076-2

Jongen, T., 1998. Simulation and Modeling of Turbulent Incompressible Flows.

Katsui, T., Asai, H., Himeno, Y., Tahara, Y., 2005. The proposal of a new friction line, in: Fifth Osaka Colloquium on Advanced CFD Applications to Ship Flow and Hull Form Design, Osaka, Japan.

Kolmogorov, A.N., 1942. Equations of turbulent motion of an incompressible fluid. Izv. Acad. Sci. USSR Phys 6, 56-58.

Kolmogorov, A.N., 1941. The local structure of turbulence in incompressible viscous fluid for very large Reynolds numbers. Cr Acad. Sci. URSS 30.

Kooij, G.L., Botchev, M.A., Frederix, E.M.A., Geurts, B.J., Horn, S., Lohse, D., van der Poel, E.P., Shishkina, O., Stevens, R.J.A.M., Verzicco, R., 2018. Comparison of computational codes for direct numerical simulations of turbulent Rayleigh-Bénard convection. Comput. Fluids 166, 1-8. https://doi.org/10.1016/j.compfluid.2018.01.010

Kornev, N., Shevchuk, I., Abbas, N., Anschau, P., Samarbakhsh, S., 2019. Potential and limitations of scale resolved simulations for ship hydrodynamics applications. Sh. Technol. Res. 7255. https://doi.org/10.1080/09377255.2019.1574965

Langtry, R.B., 2006. A Correlation-Based Transition Model using Local Variables for Unstructured Parallelized CFD codes. Univ. Stuttgart. https://doi.org/10.1115/1.2184352

Larsson, L., Stern, F., Visonneau, M., 2014. Numerical Ship Hydrodynamics: An assessment of the Gothenburg 2010 Workshop. Springer. https://doi.org/10.1007/978-94-007-7189- 
Launder, B.E., Sharma, B.I., 1974. Application of the energy-dissipation model of turbulence to the calculation of flow near a spinning disc. Lett. Heat Mass Transf. 1, 131-137. https://doi.org/10.1016/0094-4548(74)90150-7

Laurence, D., Uribe, J.C., Utyuzhnikov, S.V., 2004. A robust formulation of the v2-f model and its applications. Int. J. Numer. Methods Fluids 00, 1-6.

Lesieur, M., 2008. Turbulence in fluids, 4th ed, Physics of Fluids. Springer. https://doi.org/10.1063/1.1711203

Lien, F.S., Kalitzin, G., Durbin, P.A., 1998. RANS modeling for compressible and transitional flows. Cent. Turbul. Res. Summer Progr. 1998 1-20.

Liu, W., Demirel, Y.K., Djatmiko, E.B., Nugroho, S., Tezdogan, T., Kurt, R.E., Supomo, H., Baihaqi, I., Yuan, Z.M., Incecik, A., 2018. Bilge Keel Design for the Traditional Fishing Boats of Indonesia's East Java. Int. J. Nav. Archit. ure Ocean Eng.

Magionesi, F., Di Mascio, A., 2016. Investigation and modelling of the turbulent wall pressure fluctuations on the bulbous bow of a ship. J. Fluids Struct. 67, 219-240. https://doi.org/10.1016/j.jfluidstructs.2016.09.008

Manceau, R., 2015. Recent progress in the development of the Elliptic Blending Reynoldsstress model. Int. J. Heat Fluid Flow 51, 195-220. https://doi.org/10.1016/j.ijheatfluidflow.2014.09.002

Manceau, R., Hanjalić, K., 2002. Elliptic blending model: A new near-wall Reynolds-stress turbulence closure. Phys. Fluids 14, 744-754. https://doi.org/10.1063/1.1432693

Manceau, R., Wang, M., Laurence, D., 2001. Inhomogeneity and anisotrophy effects on the redistribution term in Reynolds-averaged Navier-Stoke modelling. J. Fluid Mech. 438, 307-338. https://doi.org/10.1017/S0022112001004451

Markatos, N.C., 1986. The mathematical modelling of turbulent flows. Appl. Math. Model. 10, 190-220. https://doi.org/https://doi.org/10.1016/0307-904X(86)90045-4

Menter, F.R., 1994. Two-equation eddy-viscosity turbulence models for engineering applications. AIAA J. 32, 1598-1605. https://doi.org/10.2514/3.12149

Menter, F.R., Smirnov, P.E., Liu, T., Avancha, R., 2015. A One-Equation Local CorrelationBased Transition Model. Flow, Turbul. Combust. 95, 583-619. https://doi.org/10.1007/s10494-015-9622-4

Michell, J.H., 1898. The wave-resistance of a ship. London, Edinburgh, Dublin Philos. Mag. J. Sci. 45, 106-123.

Molland, A.F., Turnock, S.R., Hudson, D.A., 2017. Model-Ship Extrapolation, in: Ship Resistance and Propulsion: Practical Estimation of Ship Propulsive Power. Cambridge University Press, Cambridge, pp. 70-85. https://doi.org/10.1017/9781316494196.006

Mucha, P., el Moctar, B., 2014. Numerical Prediction of Resistance and Squat for a Containership in Shallow.

Müller, C., Herbst, F., 2014. Modelling of crossflow-induced transition based on local variables, in: 6th European Conference on Computational Fluid Dynamics (ECFD). Barcelona, Spain, pp. 20-25. 
Ozdemir, Y.H., Cosgun, T., Dogrul, A., Barlas, B., 2016. A numerical application to predict the resistance and wave pattern of KRISO containership. Brodogradnja 67, 47-65.

Parneix, S., Durbin, P.A., Behnia, M., 1998. Computation of 3-D turbulent boundary layers using the V2F model. Flow, Turbul. Combust. 60, 19-46. https://doi.org/10.1023/A:1009986925097

Patel, V.C., Rodi, W., Scheuerer, G., 1984. Turbulence models for near-wall and low Reynolds number flows - A review. AIAA J. 23, 1308-1319. https://doi.org/10.2514/3.9086

Pereira, F.S., Eça, L., Vaz, G., 2017. Verification and Validation exercises for the flow around the KVLCC2 tanker at model and full-scale Reynolds numbers. Ocean Eng. 129, 133-148. https://doi.org/10.1016/j.oceaneng.2016.11.005

Pettersson Reif, B.A., 2006. Towards a nonlinear eddy-viscosity model based on elliptic relaxation. Flow, Turbul. Combust. 76, 241-256. https://doi.org/10.1007/s10494-0069013-y

Pettersson Reif, B.A., Mortensen, M., Langer, C.A., 2009. Towards sensitizing the nonlinear v 2-f model to turbulence structures. Flow, Turbul. Combust. 83, 185-203. https://doi.org/10.1007/s10494-008-9194-7

Phillips, O.M., 1955. The irrotational motion outside a free turbulent boundary. Math. Proc. Cambridge Philos. Soc. 51, 220-229. https://doi.org/10.1017/S0305004100030073

Ponkratov, D., 2016. Lloyd's Register workshop on ship scale hydrodynamics, in: Ponkratov, D. (Ed.), 2016 Workshop on Ship Scale Hydrodynamic Computer Simulation. p. 2016. https://doi.org/10.1002/ejoc.201200111

Posa, A., Broglia, R., Felli, M., Falchi, M., Balaras, E., 2019. Characterization of the wake of a submarine propeller via Large-Eddy simulation. Comput. Fluids 184, 138-152. https://doi.org/10.1016/j.compfluid.2019.03.011

Prandtl, L., 1925. Report on the investigation of developed turbulence, Translation of “ Bericht über Untersuchungen zur ausgebildeten Turbulenz." Zeitschrift für angewandte Mathematik und Mechanik, vol. 5, no. 2, April 1925.

Revell, A.J., Benhamadouche, S., Craft, T., Laurence, D., Yaqobi, K., 2005. A Stress-Strain Lag Eddy Viscosity Model for Unsteady Mean Flow. Eng. Turbul. Model. Exp. 627 , 117-126. https://doi.org/10.1016/B978-008044544-1/50010-8

Richardson, L.F., 1922. Weather prediction by numerical process, 1st ed. Cambridge University Press.

Richardson, L.F., 1911. The Approximate Arithmetical Solution by Finite Differences of Physical Problems involving Differential Equations, with an Application to the Stresses in a Masonry Dam. Philos. Trans. th R. Soc. London,Containing Pap. a Math. Phys. Character 210, 307-357.

Roache, P.J., 2016. Verification and Validation in Fluids Engineering: Some Current Issues. J. Fluids Eng. 138, 101205. https://doi.org/10.1115/1.4033979

Roache, P.J., 1998. Validation and Verification in computational science and engineering. Hermosa Albuquerque, NM. 
Roache, P.J., Ghia, K.N., White, F., 1986. Editorial Policy Statement on the Control of Numerical Accuracy. J. Fluids Eng. 108.

Robertson, E., Choudhury, V., Bhushan, S., Walters, D.K., 2015. Validation of OpenFOAM numerical methods and turbulence models for incompressible bluff body flows. Comput. Fluids 123, 122-145. https://doi.org/10.1016/j.compfluid.2015.09.010

Rotta, J., 1951. Statistical Theory of Inhomogeneous Turbulence. NASA Tech. Doc.

Roy, C., Blottner, F., 2006. Review and Assessment of Turbulence Models for Hypersonic Flows: 2D/Asymmetric Cases. 44th AIAA Aerosp. Sci. Meet. Exhib. https://doi.org/10.2514/6.2006-713

Saffman, P.G., 1970. A Model for Inhomogeneous Turbulent Flow. Proc. R. Soc. A Math. Phys. Eng. Sci. 317, 417-433. https://doi.org/10.1098/rspa.1970.0125

Saffman, P.G., Wilcox, D.C., 1974. Turbulence-Model Predictions for Turbulent Boundary Layers. AIAA J. 12, 541-546. https://doi.org/10.2514/3.49282

Saric, W.S., Reed, H.L., White, E.B., 2002. Stability and Transition of three -Dimensional Boundary Layers. Annu. Rev. Fluid Mech. 35, 413-440. https://doi.org/10.1146/annurev.fluid.35.101101.161045

Sarkar, S., Lakshmanan, B., 1991. Application of a Reynolds stress turbulence model to the compressible shear layer. AIAA J. 29, 743-749. https://doi.org/10.2514/3.10649

Schoenherr, K., 1932. Resistance of flat surfaces moving through a fluid. Trans. Soc. Nav. Arch. Mar. Eng. 40, 279-313.

Shih, T., 1990. An improved k-epsilon model for near-wall turbulence and comparison with direct numerical simulation. NASA STI/Recon Tech. Rep. N 0-21.

Shih, T.H., Liou, W.W., Shabbir, A., Yang, Z., Zhu, J., 1995. A new k- $\varepsilon$ eddy viscosity model for high reynolds number turbulent flows. Comput. Fluids 24, 227-238. https://doi.org/10.1016/0045-7930(94)00032-T

Siemens, 2018. Star-CCM+ User Guide version 13.04.

Song, S., Demirel, Y.K., Atlar, M., 2019. An investigation into the effect of biofouling on the ship hydrodynamic characteristics using CFD. Ocean Eng. 175, 122-137. https://doi.org/10.1016/j.oceaneng.2019.01.056

Spalart, P., Allmaras, S., 1992. A one-equation turbulence model for aerodynamic flows. 30th Aerosp. Sci. Meet. Exhib.

St Denis, M., Pierson, W.J., 1953. On the Motions of Ships in Confused Seas. Trans. Soc. Nav. Archit. Mar. Eng. 61, 280-357.

Starke, A.R., Drakopoulos, K., Toxopeus, S.L., Turnock, S.R., 2017. RANS-based full-scale power predictions for a general cargo vessel, and comparison with sea-trial results. 7th Int. Conf. Comput. Methods Mar. Eng. Mar. 2017 2017-May, 353-364.

Stern, F., Wilson, R., Shao, J., 2006. Quantitative V\&V of CFD simulations and certification of CFD codes. Int. J. Numer. Methods Fluids 50, 1335-1355. https://doi.org/10.1002/fld.1090

Stroh, A., 2016. Control of Spatially Developing TurbulentBoundary Layers for Skin Friction 
DragReduction, in: Nagel W., Kröner D., Resch M. (Eds) High Performance Computing in Science and Engineering '16. https://doi.org/10.1109/CAHPC.2005.27

Tahara, Y., Stern, F., 1994. Validation of an interactive approach for calculating ship boundary layers and wakes for nonzero froude number. Comput. Fluids 23, 785-816. https://doi.org/10.1016/0045-7930(94)90066-3

Terziev, M., Tezdogan, T., Incecik, A., 2019. Under Review: A geosim analysis of ship resistance decomposition and scale effects with the aid of CFD. Appl. Ocean Res.

Terziev, M., Tezdogan, T., Oguz, E., Gourlay, T., Demirel, Y.K., Incecik, A., 2018. Numerical investigation of the behaviour and performance of ships advancing through restricted shallow waters. J. Fluids Struct. 76, 185-215. https://doi.org/10.1016/j.jfluidstructs.2017.10.003

Tezdogan, T., Incecik, A., Turan, O., 2016a. Full-scale unsteady RANS simulations of vertical ship motions in shallow water. Ocean Eng. 123, 131-145. https://doi.org/10.1016/j.oceaneng.2016.06.047

Tezdogan, T., Incecik, A., Turan, O., 2016b. A numerical investigation of the squat and resistance of ships advancing through a canal using CFD. J. Mar. Sci. Technol. 21, 86101. https://doi.org/10.1007/s00773-015-0334-1

Tuck, E.O., 1967. Sinkage and Trim in Shallow Water of Finite Width. Schiffstechnik 14, 92-94.

Tuck, E.O., 1966. Shallow-Water Flows Past Slender Bodies. J. Fluid Mech. 26, 81-95. https://doi.org/10.1017/S0022112066001101

Tuck, E.O., Lazauskas, L. V, 2008. Drag on a ship and Michell's integral, in: 22nd Int. Congress of Theoretical and Applied Mechanics, Adelaide, South Australia, August.

van der Poel, E.P., Ostilla-Mónico, R., Donners, J., Verzicco, R., 2015. A pencil distributed finite difference code for strongly turbulent wall-bounded flows. Comput. Fluids 116, 10-16. https://doi.org/10.1016/j.compfluid.2015.04.007

van Driest, E.R., 1956. On Skin Friction and Heat Transfer Near the Stagnation Point, NACA Report, AL-2267.

van Strien, M., 2014. On the origins and foundations of laplacian determinism. Studies in History and Philosophy of Science Part A 45, 24-31. https://doi.org/10.1016/j.shpsa.2013.12.003

van Wijngaarden, E., 2005. Recent developments in predicting propeller-induced hull pressure pulses. Proc. 1st Int. Sh. Noise Vib. Conf. 1-8.

Velázquez, M.J.N., Asuero, A.G., 2017. Youden Two-Sample Method, in: Quality Control and Assurance-An Ancient Greek Term Re-Mastered. IntechOpen.

Wang, J., Zou, L., Wan, D., 2018. Numerical simulations of zigzag maneuver of free running ship in waves by RANS-Overset grid method. Ocean Eng. 162, 55-79.

https://doi.org/10.1016/j.oceaneng.2018.05.021

Wilcox, D.C., 2008. Formulation of the k-w Turbulence Model Revisited. AIAA J. 46, 28232838. https://doi.org/10.2514/1.36541

Wilcox, D.C., 2006. Turbulence modeling for CFD, 3rd ed, Transportation Research Record. 
DCW Industries. https://doi.org/10.1016/j.aqpro.2013.07.003

Wilcox, D.C., 1988. Reassessment of the scale-determining equation for advanced turbulence models. AIAA J. 26, 1299-1310. https://doi.org/10.2514/3.10041

Wnęk, A.D., Sutulo, S., Soares, C.G., 2018. CFD Analysis of Ship-to-Ship Hydrodynamic Interaction. J. Mar. Sci. Appl. 17, 21-37. https://doi.org/https://doi.org/10.1007/s11804018-0010-z

Wolfshtein, M., 1969. The velocity and temperature distribution in one-dimensional flow with turbulence augmentation and pressure gradient. Int. J. Heat Mass Transf. 12, 301318. https://doi.org/10.1016/0017-9310(69)90012-X

Xing, T., Stern, F., 2010. Factors of Safety for Richardson Extrapolation. J. Fluids Eng. 132, 061403. https://doi.org/10.1115/1.4001771

Youden, W.J., 1972. Graphical diagnosis of inter-laboratory test results. J. Qual. Technol. 4. https://doi.org/https://doi.org/10.1080/00224065.1972.11980509

Zeng, Q., Thill, C., Hekkenberg, R., Rotteveel, E., 2018. A modification of the ITTC57 correlation line for shallow water. J. Mar. Sci. Technol. 0, 0. https://doi.org/10.1007/s00773-018-0578-7 